\title{
Physics prospects with the second oscillation maximum at the Deep Underground Neutrino Experiment
}

\author{
Jogesh Rout $\odot,{ }^{1, *}$ Sheeba Shafaq ${ }^{1},{ }^{1, \dagger}$ Mary Bishai ${ }^{2, \dagger}$ and Poonam Mehta $\oplus^{1, \S}$ \\ ${ }^{1}$ School of Physical Sciences, Jawaharlal Nehru University, New Delhi 110067, India \\ ${ }^{2}$ Brookhaven National Laboratory, P.O. Box 5000, Upton, New York 11973-5000, USA
}

(Received 19 February 2021; accepted 16 April 2021; published 2 June 2021)

\begin{abstract}
Current long-baseline neutrino-oscillation experiments such as $\mathrm{NO} \nu \mathrm{A}$ and $\mathrm{T} 2 \mathrm{~K}$ are mainly sensitive to physics in the neighborhood of the first oscillation maximum of the $\nu_{\mu} \rightarrow \nu_{e}$ oscillation probability. The future Deep Underground Neutrino Experiment (DUNE) utilizes a wide-band beam tune optimized for $C P$-violation sensitivity that fully covers the region of the first maxima and part of the second. In the present study, we elucidate the role of second oscillation maximum in addressing issues pertaining to unknowns in the standard three-flavor paradigm. We consider a new DUNE beam tune optimized for coverage of the region of the second oscillation maxima which could be realized using proposed accelerator upgrades that provide multimegawatts of power at proton energies of $8 \mathrm{GeV}$. We find that the addition of the multimegawatt $8 \mathrm{GeV}$ beam to DUNE wide-band running leads to modest improvement in sensitivity to $C P$ violation, mass hierarchy, and the octant of $\theta_{23}$ as well as the resolution of $\delta$ and the Jarlskog invariant. Significant improvements to the DUNE neutrino energy resolution yield a much larger improvement in performance. We conclude that the standard DUNE wide-band beam when coupled with excellent detector resolution capabilities is sufficient to resolve $\delta$ to better than $\sim 12^{\circ}$ for all values of $\delta$ in a decade of running. For second maxima ( $8 \mathrm{GeV}, 3 \mathrm{MW}$ ) beam running concurrently with the standard wide-band $(80 \mathrm{GeV}$, $2.2 \mathrm{MW}$ ) beam for five of the 10 years, it is found that $\delta$ can be further resolved better than $\sim 10^{\circ}$ for all values of $\delta$.
\end{abstract}

DOI: $10.1103 /$ PhysRevD.103.116003

\section{INTRODUCTION}

Pontecorvo's original insight that neutrinos oscillate among one another [1] has been confirmed by a variety of neutrino-oscillation experiments involving a wide range of energies and baselines. The idea of neutrino oscillations among the three light active neutrino flavors has been rewarded with a Nobel prize in 2015 [2]. The parameters entering the neutrino-oscillation framework have been measured to a fairly good precision (see the recent global fit analyses $[3,4])$. The best-fit values and $3 \sigma$ range of neutrino mass and mixings deciphered from oscillation data are given in Table I. Yet, there are some open questions in the standard mass-induced oscillation framework. These include the question of neutrino mass hierarchy (sign of

\footnotetext{
jogesh.rout1@gmail.com

sheebakhawaja7@gmail.com

mbishai@bnl.gov

${ }^{\S}$ pm@jnu.ac.in
}

Published by the American Physical Society under the terms of the Creative Commons Attribution 4.0 International license. Further distribution of this work must maintain attribution to the author(s) and the published article's title, journal citation, and DOI. Funded by SCOAP ${ }^{3}$.
$\left.\Delta m_{31}^{2}\right)$, the value of the $C P$-violating phase $(\delta)$ and determining the correct octant of $\theta_{23}$. Furthermore, it is desirable to improve the precision measurements of the parameters entering the oscillation framework.

Determination of neutrino mass hierarchy $(\mathrm{MH})$ would allow us to get closer toward determining the underlying structure of the neutrino mass matrix by being able to discriminate between theoretical models giving rise to neutrino masses [5]. Along with the $C P$-violating phase $\delta$, it impacts the effectiveness of the leptogenesis scenario which can explain the matter-antimatter asymmetry of the Universe [6].

The next-generation neutrino-oscillation experiments would allow us to precisely determine the known parameters and determine the remaining unknowns in the neutrino-oscillation formalism. The long-baseline neutrino experiments are designed such that the desirable physics outcome is achieved. Typically, the optimal combination is for a value of baseline $(L)$ and energy $(E)$ for which $P_{\mu e}$ has its first peak. This is referred to as the first oscillation maximum. Typically, for shorter baselines, the higher oscillation maxima are unaccessible, as the energies at which these occur are low and difficult to produce experimentally. For longer baselines, it may be possible to access the information from the second (and higher) 
TABLE I. Standard oscillation parameters and their uncertainties used in our study. The values were taken from the global fit analysis in Ref. [3]. If the $3 \sigma$ upper and lower limit of a parameter is $x_{u}$ and $x_{l}$, respectively, the $3 \sigma$ uncertainty is $\left(x_{u}-x_{l}\right) /\left(x_{u}+x_{l}\right) \%$. Note that $\mathrm{NH}$ stands for normal hierarchy and $\mathrm{IH}$ for inverted hierarchy.

\begin{tabular}{lccc}
\hline \hline Parameter & Best-fit value & $3 \sigma$ range & $3 \sigma$ uncertainty \\
\hline$\theta_{12}\left[^{\circ}\right]$ & 34.3 & $31.4-37.4$ & 8.72 \\
$\theta_{13}(\mathrm{NH})\left[^{\circ}\right]$ & 8.58 & $8.16-8.94$ & 4.56 \\
$\theta_{13}(\mathrm{IH})\left[^{\circ}\right]$ & 8.63 & $8.21-8.99$ & 4.53 \\
$\theta_{23}(\mathrm{NH})\left[^{\circ}\right]$ & 48.8 & $41.63-51.32$ & 10.42 \\
$\theta_{23}(\mathrm{IH})\left[^{\circ}\right]$ & 48.8 & $41.88-51.30$ & 10.11 \\
$\Delta m_{21}^{2}\left[\mathrm{eV}^{2}\right]$ & $7.5 \times 10^{-5}$ & {$[6.94-8.14] \times 10^{-5}$} & 8.0 \\
$\Delta m_{31}^{2}(\mathrm{NH})\left[\mathrm{eV}^{2}\right]$ & $+2.56 \times 10^{-3}$ & $-[2.37-2.55] \times 10^{-3}$ & 3.72 \\
$\Delta m_{31}^{2}(\mathrm{IH})\left[\mathrm{eV}^{2}\right]$ & $-2.46 \times 10^{-3}$ & {$[-\pi, 0] \cup[0.8 \pi, \pi]$} & 3.7 \\
$\delta(\mathrm{NH})[\mathrm{rad}]$ & $-0.8 \pi$ & {$[-0.86 \pi,-0.1 \pi]$} & $\ldots$ \\
$\delta(\mathrm{IH})[\mathrm{rad}]$ & $-0.46 \pi$ & & $\ldots$ \\
\hline \hline
\end{tabular}

oscillation maxima. At the second oscillation maximum, one expects higher sensitivity to $\delta$, as the size of the $\delta$-dependent interference term is a factor of $\sim 3$ larger than that at the first oscillation maximum.

A promising future experiment is the Deep Underground Neutrino Experiment (DUNE). A high-purity muon neutrino beam will be produced at Fermilab and will travel $1300 \mathrm{~km}$ to a liquid argon (LAr) far detector placed at an on-axis location at Sanford Underground Research Facility. The primary aim of DUNE is to address the question of $C P$ violation and identify the neutrino mass hierarchy [7-9]. A wide-band neutrino beam originating from the Fermilab proton complex is considered for DUNE. A systematic evaluation of optimal baseline for discovery of $C P$ violation, determination of the mass hierarchy, and resolution of the $\theta_{23}$ octant in a long-baseline oscillation experiment was carried out by Bass et al. [10], and it was concluded that, for achieving unambiguous measurement of these parameters, one needed a baseline at least of the order of $1000 \mathrm{~km}$. It was further shown from the asymmetry plot that $C P$ measurement was better achieved in the vicinity of the second oscillation maximum irrespective of the mass hierarchy, and results for sensitivities to standard threeflavor oscillation parameters were presented. The authors had considered two detector types-water Cherenkov and LAr-and performed the study for the erstwhile Long Baseline Neutrino Experiment (LBNE).

The idea of utilizing the second oscillation maximum in neutrino experiments is not new. The prospect of using a high-intensity low-energy neutrino beam using Project X was studied in Ref. [11], and it was demonstrated that the simultaneous operation of 8 and $60 \mathrm{GeV}$ beams in conjunction with a water Cherenkov detector allows for sensitivity to $\nu_{\mu} \rightarrow \nu_{e}$ oscillation at the second oscillation maximum. The focus of the study was to attain highprecision measurement of $\theta_{13}$ and $\delta$. With the goal of enhancing the mass hierarchy sensitivity, the authors of Ref. [12] introduced a second detector at an off-axis location (the same beam was used) and obtained marginal improvement for certain values of $\delta$ in the worse half plane of $\delta$ values. In Ref. [13], the idea of utilizing different oscillation maxima was invoked in discussing the role and interplay between appearance and disappearance channels at long-baseline experiments for precision measurement of $\delta$ and $\theta_{23}$. In Ref. [14], for the considered experimental setup (Fermilab to Deep Underground Science and Engineering Laboratory LBNE), it was concluded that the second maximum plays only a marginal role due to the experimental difficulties to obtain a statistically significant and sufficiently background-free event sample at low energies. The impact of improved neutrino energy reconstruction capabilities at DUNE has been studied in Ref. [15]. The European Spallation Source (ESS) neutrino Super Beam (ESS $2 \mathrm{SB})$ facility in Europe utilizes the second oscillation peak to maximize the discovery potential to leptonic $C P$ violation with the detector placed at $L=540 \mathrm{~km}$. The $L / E$ is such that the second oscillation peak can naturally be exploited in order to measure oscillation parameters [16-22]. Among other experiments, the MuOn-decay MEdium baseline NeuTrino beam facility (MOMENT) [23] proposal $(L=150 \mathrm{~km})$ has similar $L / E$ as the $\mathrm{ESS} L \mathrm{SB}$. The prospect of precision measurement of $\delta$ at MOMENT has been studied in Ref. [24]. The Tokai-toHyper-Kamiokande-to-Korea (T2HKK) [25] proposal, in which the first and the second oscillation maxima are measured with two detectors located at different sites, will have the same $L / E$ range. More recently, invisible neutrino decay at ESS $\nu \mathrm{SB}$ has been explored in Ref. [26] and a comparative analysis of T2HK, T2HKK, and ESS $\nu$ SB in the context of neutrino decay has been carried out in Ref. [27].

However, it should be noted that a comprehensive and detailed assessment of the role of different oscillation maxima for a long-baseline experiment such as DUNE has not been addressed in the earlier work, and this is the main motivation of the present study. In the present article, we begin with a probability level analysis highlighting the role of second oscillation maxima and its impact on the current unknowns in neutrino-oscillation physics. We also 
address the issue of extraction of intrinsic $C P$ violation using the two beams and show that the second oscillation maximum helps in resolving the ambiguity in $C P$ phase measurement. We utilize optimal beam tunes to explore the precise role of first and second oscillation maxima. The standard beam tune used in almost all the studies connected with DUNE sensitivities is derived from an $80 \mathrm{GeV}$ proton beam energy, and this is well suited to study oscillations in the vicinity of the first oscillation maximum. The wide-band default DUNE beam also covers a portion of the region of the second oscillation maxima. However, one needs a different source to fully utilize the second oscillation maximum-here, we employ an $8 \mathrm{GeV}$ beam as was proposed in previous Project $X$ studies [11] to harness the signal at second oscillation maximum. We note that the Project X $8 \mathrm{GeV}$ multimegawatt beam could be realized by the proposed Fermilab PIP-III accelerator upgrade option utilizing a 6-8 GeV pulsed superconducting rf (SRF) linac [28,29]. The PIP-III pulsed SRF linac could generate $4 \mathrm{MW}$ of power at $8 \mathrm{GeV}$, of which only a few hundred kilowatts would be needed to increase the power from the Main Injector accelerator to $\sim 2 \mathrm{MW}$ at $80 \mathrm{GeV}$. Therefore, it may be possible to utilize a Main Injector beam of $\sim 2 \mathrm{MW}$ at $80 \mathrm{GeV}$ simultaneously with an $\sim 3 \mathrm{MW}$ at $8 \mathrm{GeV}$ beam derived from the SRF linac to generate neutrino beams for DUNE.
The present article is structured as follows. In Sec. II, we describe the basic framework used in the present work. This includes a review of electron neutrino appearance probability in vacuum and in matter, oscillation maxima of $P_{\mu e}, C P$ asymmetry, $\mathrm{MH}$ asymmetry, and a description of an observable for separation of intrinsic versus extrinsic contribution to the $C P$ phase. In Sec. III, we describe the experimental inputs such as beam tunes used and detector details. Section IV is devoted to a discussion at the level of event rates (with a discussion on backgrounds) at DUNE using different beam tunes. In Sec. VI, we present our main results for sensitivities to $C P$, mass hierarchy, octant of $\theta_{23}$, resolution of $\delta$, and $1 \sigma$ contours from a two-dimensional fit to $\theta_{23}$ and $\delta$ at the level of $\chi^{2}$. We summarize our outcome in Sec. VII.

\section{FRAMEWORK}

Neutrino oscillations have their origin in the nonzero neutrino masses and mixing among the neutrino flavors. The standard paradigm of neutrino oscillations involves three flavors of neutrinos which are superpositions of the mass states carrying well-defined masses. The mixing matrix $(\mathcal{U})$ in the Pontecorvo-Maki-Nakagawa-Sakata (PMNS) parametrization [30] is given by

$$
\mathcal{U} \equiv \mathcal{U}_{\mathrm{PMNS}}=\left(\begin{array}{ccc}
1 & 0 & 0 \\
0 & c_{23} & s_{23} \\
0 & -s_{23} & c_{23}
\end{array}\right)\left(\begin{array}{ccc}
c_{13} & 0 & s_{13} e^{-i \delta} \\
0 & 1 & 0 \\
-s_{13} e^{i \delta} & 0 & c_{13}
\end{array}\right)\left(\begin{array}{ccc}
c_{12} & s_{12} & 0 \\
-s_{12} & c_{12} & 0 \\
0 & 0 & 1
\end{array}\right)
$$

where $s_{i j}=\sin \theta_{i j}, c_{i j}=\cos \theta_{i j}$, and $\delta$ is the Dirac-type $C P$ phase. Additionally, if neutrinos are Majorana, there can be two additional Majorana-type phases in the threeflavor case. However, those Majorana phases play no role in neutrino oscillations [31].

In a typical long-baseline experiment such as DUNE, at the source, a high-purity beam of muon neutrinos ( $>90 \%$ pure) is produced via pion and kaon decays. In principle, all the oscillation channels $\nu_{\mu} \rightarrow \nu_{e}\left(\nu_{e}\right.$ appearance), $\nu_{\mu} \rightarrow \nu_{\mu}$ ( $\nu_{\mu}$ disappearance), and $\nu_{\mu} \rightarrow \nu_{\tau}\left(\nu_{\tau}\right.$ appearance) should be accessible at DUNE. However, with the standard beam tune of the LBNF beam line, the $\nu_{\mu} \rightarrow \nu_{\tau}$ appearance channel does not lead to sizable event samples [32], and it is not possible to probe this channel. In recent studies, it has been shown that higher-energy beam tunes may prove useful to probe this channel [32-34]. However, here, we are concerned with the standard beam tune at DUNE and the flux relevant for the second maxima, so the channels considered are the $\nu_{e}$ appearance channel and $\nu_{\mu}$ disappearance channel. In order to have a clear understanding of the role of second oscillation maximum vis-à-vis the first oscillation maximum in probing the current unknowns, we briefly describe the main features of the oscillation probability for the $\nu_{\mu} \rightarrow \nu_{e}$ channel.

\section{A. Brief review of $\boldsymbol{P}_{\boldsymbol{\mu}}$ in vacuum and in matter}

For propagation of neutrinos through vacuum, $P_{\mu e}^{v}$ can be expressed as (see [35])

$$
P_{\mu e}^{v} \simeq P_{0}^{v}+P_{\sin \delta}^{v}+P_{\cos \delta}^{v}+P_{3}^{v},
$$

where

$$
\begin{gathered}
P_{0}^{v}=\sin ^{2} \theta_{23} \sin ^{2} 2 \theta_{13} \sin ^{2} \Delta_{31}, \\
P_{\sin \delta}^{v}=\sin \delta \cos \theta_{13} \sin 2 \theta_{12} \sin 2 \theta_{13} \sin 2 \theta_{23} \\
\times \sin ^{2} \Delta_{31} \sin \left(2 \alpha \Delta_{31}\right), \\
P_{\cos \delta}^{v}=\cos \delta \cos \theta_{13} \sin 2 \theta_{12} \sin 2 \theta_{13} \sin 2 \theta_{23} \sin \Delta_{31} \\
\times \sin \left(2 \alpha \Delta_{31}\right) \cos \Delta_{31}, \\
P_{3}^{v}=\cos ^{2} \theta_{23} \sin ^{2} 2 \theta_{12} \sin ^{2}\left(\alpha \Delta_{31}\right) .
\end{gathered}
$$

Here, $\Delta_{31}=\Delta m_{31}^{2} L / 4 E$ and $\alpha=\Delta m_{21}^{2} / \Delta m_{31}^{2} . E$ is the neutrino energy in $\mathrm{GeV}$, and $L$ is the baseline in kilometers. For antineutrinos, $\delta \rightarrow-\delta$. Here, $P_{0}^{v}$ is the dominant term [Eq. (3)]. The $C P$ phase dependence lies in two interference 
terms, $P_{\sin \delta}^{v}$ and $P_{\cos \delta}^{v}$ [see Eqs. (3b) and (3c)]. $P_{\sin \delta}^{v}$ is the $C P$-violating term, as it changes sign for antineutrinos, while the $P_{\cos \delta}^{v}$ is the $C P$-conserving term. It is clear that both $\Delta m_{21}^{2}$ and $\Delta m_{31}^{2}$ are required to be nonzero in order to extract any information about the $C P$ phase. $P_{3}^{v}$ is a relatively smaller term [Eq. (3d)].

In the presence of standard interactions with matter, Eqs. (2) and (3) are no longer applicable, and it is tedious to obtain approximate analytic expressions. It becomes imperative to use certain approximations to derive an analytical expression for $P_{\mu e}^{m}$. One typically uses constant density approximation and also the fact that $\alpha$ and $\theta_{13}$ are small parameters so that one can employ perturbation techniques to obtain an expression (valid up to second order) for $P_{\mu e}^{m}$ (see [36-38]). Here again, we can write down the approximate expression for probability as a sum of four terms which are modified in the presence of matter:

$$
P_{\mu e}^{m} \simeq P_{0}^{m}+P_{\sin \delta}^{m}+P_{\cos \delta}^{m}+P_{3}^{m},
$$

where

$$
\begin{aligned}
P_{0}^{m}=\sin ^{2} \theta_{23} \frac{\sin ^{2} 2 \theta_{13}}{(\hat{A}-1)^{2}} \sin ^{2}\left((\hat{A}-1) \Delta_{31}\right), \\
P_{\sin \delta}^{m}=\alpha \frac{\sin \delta \cos \theta_{13} \sin 2 \theta_{12} \sin 2 \theta_{13} \sin 2 \theta_{23}}{\hat{A}(1-\hat{A})} \\
\times \sin \left(\Delta_{31}\right) \sin \left(\hat{A} \Delta_{31}\right) \sin \left((1-\hat{A}) \Delta_{31}\right), \\
P_{\cos \delta}^{m}=\alpha \frac{\cos \delta \cos \theta_{13} \sin 2 \theta_{12} \sin 2 \theta_{13} \sin 2 \theta_{23}}{\hat{A}(1-\hat{A})} \\
\times \cos \left(\Delta_{31}\right) \sin \left(\hat{A} \Delta_{31}\right) \sin \left((1-\hat{A}) \Delta_{31}\right), \\
P_{3}^{m}=\alpha^{2} \frac{\cos ^{2} \theta_{23} \sin ^{2} 2 \theta_{12}}{\hat{A}^{2}} \sin ^{2}\left(\hat{A} \Delta_{31}\right),
\end{aligned}
$$

where $\hat{A}=A / \Delta m_{31}^{2}$, where $A=2 V E=2 \sqrt{2} G_{F} n_{e} E=$ $2 \times 0.76 \times 10^{-4} \times Y_{e} \times\left[\rho / \mathrm{g} \mathrm{cm}^{-3}\right] \times[E / \mathrm{GeV}] \mathrm{eV}^{2}$ is the Wolfenstein term. $G_{F}$ is the Fermi constant, and $n_{e}$ is the electron number density in Earth matter $\left(n_{e}=N_{A v o} Y_{e} \rho\right.$, where $N_{A v o}$ is the Avogadro number, $Y_{e}$ is the electron fraction, and $\rho$ is Earth matter density). For antineutrinos, $\delta \rightarrow-\delta$ and $A \rightarrow-A$. The above expression is valid only when $\alpha \Delta_{31} \lesssim 1$, which, in turn, means that the expression would give reasonable results for $E>0.8 \mathrm{GeV}$ for baselines below $8000 \mathrm{~km}$ [37].

Note that $P_{0}^{m}$ is the dominant term, as it is independent of $\alpha$ [Eq. (5a)]. We note that the $\delta$-dependent interference terms [Eqs. (5b) and (5c)] are suppressed by the hierarchy parameter $\alpha$ and the last term $P_{3}^{m}$ is proportional to $\alpha^{2}$ [Eq. (5d)].

\section{B. First and second oscillation maxima of $\boldsymbol{P}_{\mu e}$}

Since the leading term depends on $\Delta_{31}$, the physical characteristics of an appearance experiment are therefore determined by the baseline $(L)$ and neutrino energy $(E)$ at which the mixing between the $\nu_{1}$ and $\nu_{3}$ states is maximal. If we look at the leading oscillatory term of $P_{\mu e}^{v}$, we get oscillation maxima at

$$
\begin{aligned}
\frac{\Delta m_{31}^{2} L}{4 E} & =(2 n-1) \frac{\pi}{2} \\
\frac{L}{E} & =(2 n-1) \frac{\pi}{2}\left(\frac{1}{1.267}\right)\left(\frac{2.56 \times 10^{-3} \mathrm{eV}^{2}}{\Delta m_{31}^{2}}\right) \\
\Rightarrow \frac{L}{E} & \simeq(2 n-1) \times 500 \frac{\mathrm{km}}{\mathrm{GeV}},
\end{aligned}
$$

where $n$ is an integer and $n=1,2, \ldots$ stands for first, second, $\ldots$ oscillation maxima occurring at $L / E \simeq$ $500,1500, \ldots \mathrm{km} / \mathrm{GeV}$ and so on. For a fixed baseline of $1300 \mathrm{~km}$, this would imply $E^{\mathrm{I}} \simeq 2.6 \mathrm{GeV}$ and $E^{\mathrm{II}} \simeq$ $0.87 \mathrm{GeV}$ for first and second oscillation maxima, respectively. It may be possible to observe the higher $(n>1)$ oscillation maxima when the baselines are comparatively longer (so that the energies at which higher maxima occur are not too small). A useful observable to understand the impact of $C P$ phase on the oscillation probabilities [Eqs. (3) and (5)] is the $C P$ asymmetry [39], and in the next subsection we examine the analytic expression for the $C P$ asymmetry in vacuum and in matter.

\section{C. $C P$ asymmetry in vacuum and in matter}

The $C P$ asymmetry is given by

$$
A_{\mu e}^{C P}=\frac{P_{\mu e}(\delta)-\bar{P}_{\mu e}(\delta)}{P_{\mu e}(\delta)+\bar{P}_{\mu e}(\delta)}=\frac{\Delta P_{\mu e}^{C P}}{\sum P_{\mu e}^{C P}} .
$$

Since $C P$ asymmetry is a ratio of probabilities, the systematic uncertainties mostly cancel out at the level of event rates. In vacuum, the numerator is given by ${ }^{1}$

$$
\begin{aligned}
\Delta P_{\mu e}^{C P} & =8 J\left[\sin \left(2 \alpha \Delta_{31}\right) \sin ^{2}\left(\Delta_{31}\right)-\sin \left(2 \Delta_{31}\right) \sin ^{2}\left(\alpha \Delta_{31}\right)\right] \\
& =\underbrace{16 \sin \delta J_{r}\left[\sin \left(\Delta_{31}\right) \sin \left(\alpha \Delta_{31}\right) \sin (1-\alpha) \Delta_{31}\right]}_{\text {intrinsic }},
\end{aligned}
$$

where

$$
\begin{aligned}
J & =\operatorname{Im}\left(U_{\ell^{\prime} j} U_{\ell j}^{\star} U_{\ell j^{\prime}}^{\star} U_{\ell^{\prime} j^{\prime}}\right) \quad \text { where } l \neq l^{\prime} \quad \text { and } \quad j \neq j^{\prime} \\
& =\sin \theta_{12} \cos \theta_{12} \sin \theta_{23} \cos \theta_{23} \sin \theta_{13} \cos ^{2} \theta_{13} \sin \delta
\end{aligned}
$$

using $\mathcal{U} \equiv \mathcal{U}_{\text {PMNS }}$

\footnotetext{
${ }^{1}$ Equation (8) is obtained by using the exact three-flavor formula for $P_{\mu e}$ in vacuum given in Ref. [40].
} 
is the Jarlskog factor [41,42]. As a consequence of the orthogonality of any pair of different rows or columns of the mixing matrix, this imaginary part on the right side of Eq. (9) is unique and can differ at most by a sign. In vacuum, therefore, if the $C P$ phase $\delta$ is either 0 or $\pi$, the $C P$ asymmetry vanishes. However, matter effects can create a fake $C P$ asymmetry. In the case of matter, we have

$$
\begin{aligned}
\Delta P_{\mu e}^{C P} \approx & \underbrace{\sin ^{2} \theta_{23} \sin ^{2} 2 \theta_{13} \Theta_{+} \Theta_{-}}_{\text {extrinsic }} \\
& +\underbrace{8 \alpha J_{r} \frac{\sin \left(\hat{A} \Delta_{31}\right)}{\hat{A}}\left[\cos \delta \Theta_{-} \cos \Delta_{31}+\sin \delta \Theta_{+} \sin \Delta_{31}\right]}_{\text {intrinsic and extrinsic }},
\end{aligned}
$$

where $\Theta_{ \pm}=\sin \left[(\hat{A}-1) \Delta_{31}\right] /(\hat{A}-1) \pm \sin \left[(\hat{A}+1) \Delta_{31}\right] /$ $(\hat{A}+1)$ and $J_{r}=J / \sin \delta$. The presence of the first term implies that one will have a nonvanishing contribution to $\Delta P_{\mu e}^{C P}$ due to the Mikheyev-Smirnov-Wolfenstein matter effect $[43,44]$, since $\hat{A} \rightarrow-\hat{A}$ for antineutrinos. From Eq. (10), we note that the $\cos \delta$-dependent term is proportional to $\cos \Delta_{31}$. This implies that, at the location of oscillation maxima $\left(\cos \Delta_{31}=0\right)$, the $\cos \delta$ term vanishes.

In Fig. 1, we plot the probability difference as a function of $\delta$ for a fixed value of energy corresponding to first and second oscillation maxima and a baseline of $1300 \mathrm{~km}$ (for DUNE). The curves are plotted in vacuum and in matter [see Eqs. (8) and (10)]. It should be noted that all figures are obtained using the General Long Baseline Experiment Simulator (GLoBES) software [45,46], which numerically solves the full three-flavor neutrino propagation equations with the preliminary reference Earth model (PREM) [47] density profile of Earth for the values of neutrino parameters listed in Table I. The analytic expressions aid in our understanding of the salient features of the considered observables. The following comments are in order.

(i) From Eq. (8), we note that $\Delta P_{\mu e}^{C P} \propto \sin \delta$ in vacuum. While there is a $\cos \delta$ term in the presence of matter [Eq. (10)], it vanishes (at the location of first and second oscillation maxima as $\cos \Delta_{31} \rightarrow 0$ ), and $\delta$ dependence is via the $\sin \delta$ term in matter as well.

(ii) $\Delta P_{\mu e}^{C P}$ at $E^{\mathrm{II}}>\Delta P_{\mu e}^{C P}$ at $E^{\mathrm{I}}$ both in vacuum and in matter. The largest difference occurs around $\delta= \pm 90^{\circ}$. At $\delta=-90^{\circ}$, in vacuum, $\Delta P_{\mu e}^{C P} \simeq$ 0.025 at $E^{\mathrm{I}} ; \Delta P_{\mu e}^{C P} \simeq 0.075$ at $E^{\mathrm{II}}$. In matter (for $\mathrm{NH}), \Delta P_{\mu e}^{C P} \simeq 0.07$ at $E^{\mathrm{I}}$ and $\Delta P_{\mu e}^{C P} \simeq 0.088$ at $E^{\mathrm{II}}$.

(iii) For $C P$-conserving values, i.e., $\delta=0^{\circ}$ or $180^{\circ}$, in vacuum $\Delta P_{\mu e}^{C P}$ vanishes at $E^{\mathrm{I}}$ and $E^{\mathrm{II}}$. But, in matter (for $\mathrm{NH}$ ), $\Delta P_{\mu e}^{C P} \neq 0$ due to matter effects (pure extrinsic) and, in fact, $\Delta P_{\mu e}^{C P} \simeq 0.043$ at $E^{\mathrm{I}}$ and $\Delta P_{\mu e}^{C P} \simeq 0.017$ at $E^{\mathrm{II}}$.

(iv) In general, when $\delta \neq 0$, the extrinsic effects complicate the determination of the intrinsic $C P$ phase. As matter effects are more pronounced at $E^{\mathrm{I}}$ than at $E^{\mathrm{II}}$, the intrinsic versus extrinsic separation is harder at $E^{\mathrm{I}}$ than at $E^{\mathrm{II}}$.

(v) The main advantage of studies at the second maximum over the first maximum lies in our ability to extract the intrinsic $C P$ component better than that with the first maximum. This is due to the fact that $C P$ asymmetry in vacuum is larger while the matter effects are not as large.

In order to explore the features of the second oscillation maximum versus the first oscillation maximum as a function of $E$ and $L$, we show oscillograms of $C P$ asymmetry in the plane of $E$ and $L$ in Fig. 2. The location of first and second oscillation maxima in the plane of $E$ and $L$ is depicted in the plots. For $\delta=0$ (left panel), the $C P$

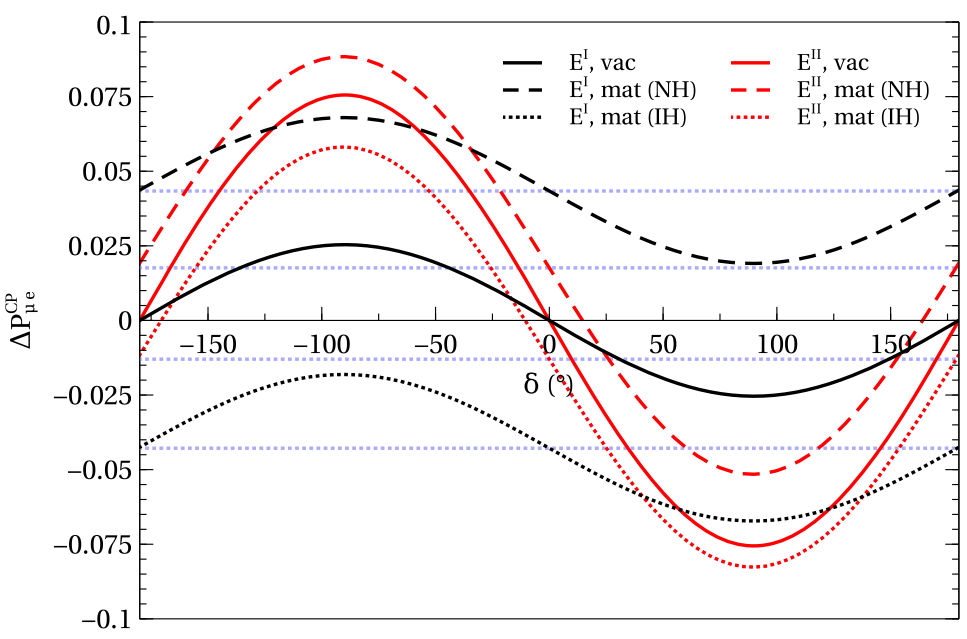

FIG. 1. $\Delta P_{\mu e}^{C P}$ plotted in vacuum (solid lines) and in matter (dashed lines for NH and dotted lines for IH) as a function of $\delta$ for a fixed baseline of $1300 \mathrm{~km}$. $E^{\mathrm{I}} \simeq 2.6 \mathrm{GeV}$ and $E^{\mathrm{II}} \simeq 0.87 \mathrm{GeV}$ refer to the first (black lines) and second (red lines) oscillation maxima, respectively. 

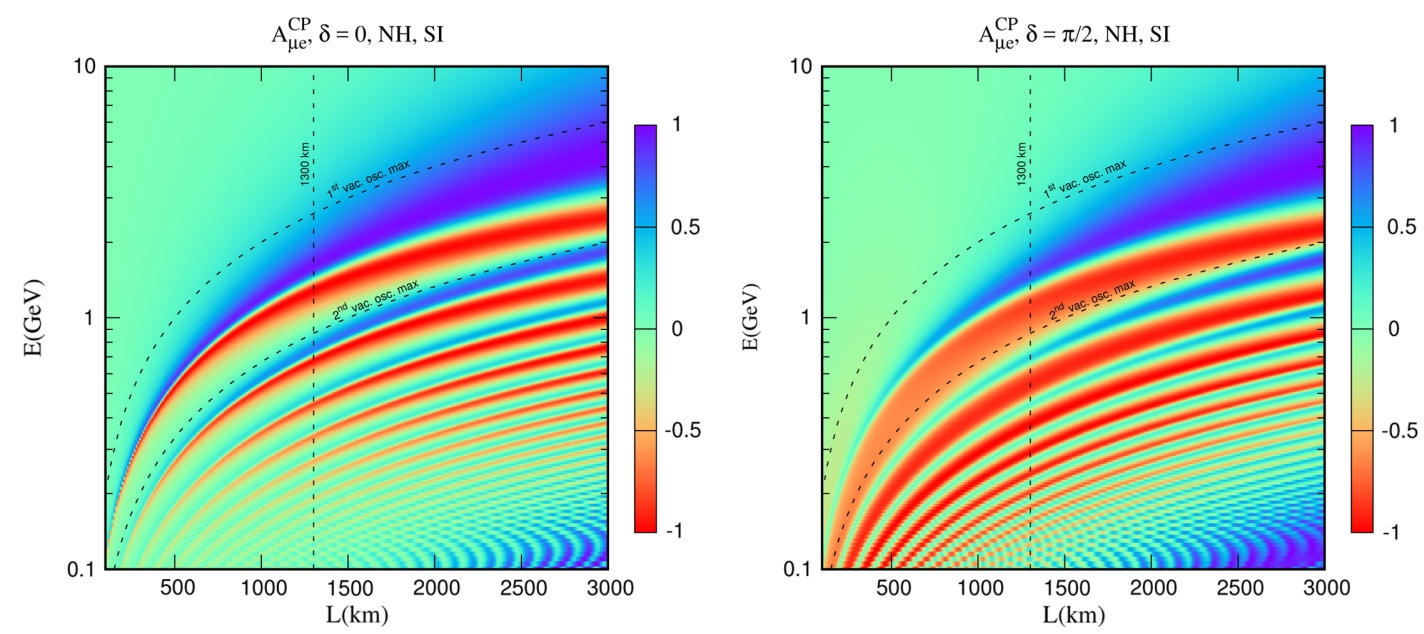

FIG. 2. Oscillogram of $C P$ asymmetry in the plane of $E$ and $L$ for $\delta=0$ and $\delta=\pi / 2$.

asymmetry at the second maximum is negligible, as expected. However, matter effects play a crucial role at the first oscillation maximum as can be seen for baselines beyond $L \approx 1000 \mathrm{~km}$, and the $C P$ asymmetry grows with $L$. For $\delta=\pi / 2$ (right panel), one can see significant change in the $C P$ asymmetry at the second oscillation maximumthis is due to the (maximal) intrinsic $C P$ contribution. The pattern of $C P$ asymmetry in the neighborhood of the first maximum is also modified due to the interplay between the intrinsic and extrinsic effects. The $C P$ asymmetry has opposite sign (red) at the second maximum in comparison to the $C P$ asymmetry at the first maximum (blue). This is consistent with Fig. 1 near $\delta \simeq \pi / 2$ for $1300 \mathrm{~km}$.

\section{Mass hierarchy asymmetry in vacuum and in matter}

The $\mathrm{MH}$ asymmetry is given by

$$
A_{\mu e}^{\mathrm{MH}}=\frac{P_{\mu e}^{\mathrm{NH}}-P_{\mu e}^{\mathrm{IH}}}{P_{\mu e}^{\mathrm{NH}}+P_{\mu e}^{\mathrm{IH}}}=\frac{\Delta P_{\mu e}^{\mathrm{MH}}}{\sum P_{\mu e}^{\mathrm{MH}}} .
$$

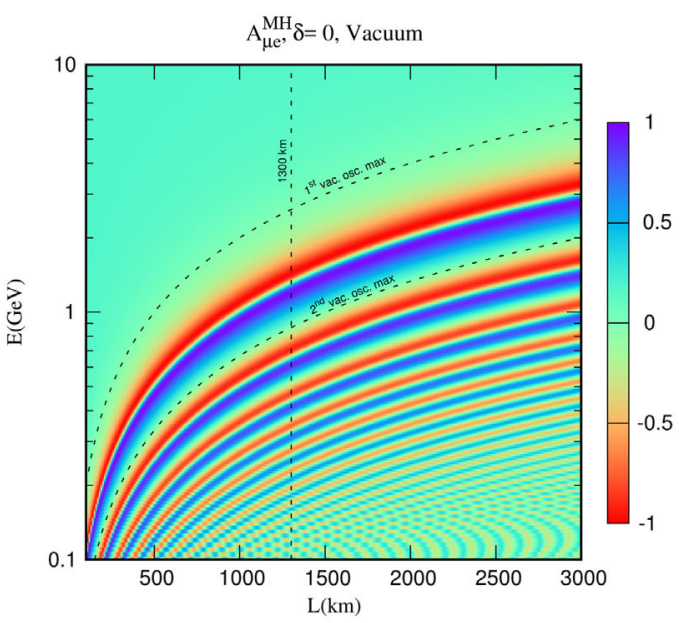

In vacuum,

$$
\begin{aligned}
\Delta P_{\mu e}^{\mathrm{MH}} \approx & 2 J_{r} \sin \Delta_{31} \sin \Delta_{21} \cos \Delta_{31} \\
& \times\left(\cos \delta \cos \Delta_{21}-8 \sin \delta \sin \Delta_{21}\right) .
\end{aligned}
$$

In matter,

$$
\begin{aligned}
\Delta P_{\mu e}^{\mathrm{MH}} \approx & \sin ^{2} \theta_{23} \sin ^{2} 2 \theta_{13} \Theta_{+} \Theta_{-} \\
& +8 \alpha J_{r} \frac{\sin \left(\hat{A} \Delta_{31}\right)}{\hat{A}}\left[\cos \delta \Theta_{+} \cos \Delta_{31}+\sin \delta \Theta_{-} \sin \Delta_{31}\right],
\end{aligned}
$$

where $\Theta_{ \pm}=\sin \left[(\hat{A}-1) \Delta_{31}\right] /(\hat{A}-1) \pm \sin \left[(\hat{A}+1) \Delta_{31}\right] /$ $(\hat{A}+1)$ as given before.

In Fig. 3, we show oscillograms for the MH asymmetry for $\delta=0$ in the plane of $E$ and $L$ for vacuum (left) and matter (right). The $\Delta P_{\mu e}^{\mathrm{MH}}$ is expected to vanish at all nodes (maxima and minima), since $\Delta P_{\mu e}^{\mathrm{MH}} \propto \cos \Delta_{31} \sin \Delta_{31}$ in vacuum [Eq. (12)]. This is independent of the value of

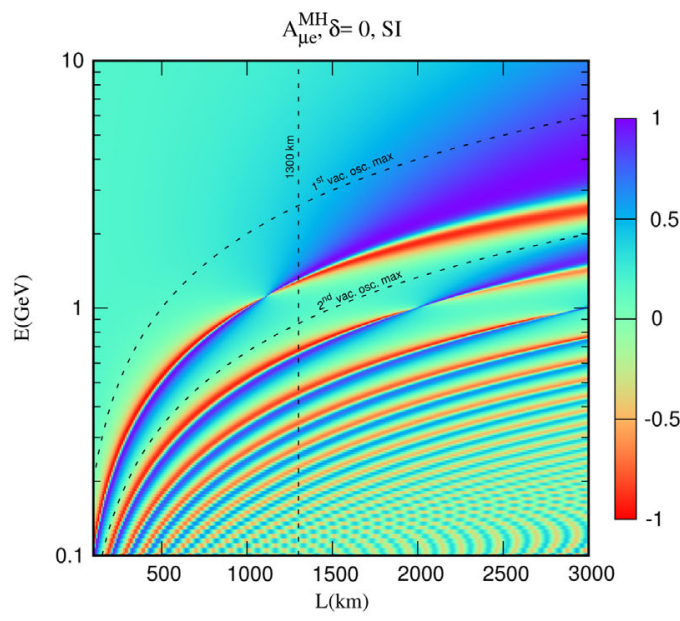

FIG. 3. Oscillogram of MH asymmetry in the plane of $E$ and $L$ for $\delta=0$ in vacuum (left) and matter (right). 
$\delta$ (Fig. 3, left panel). From the left panel in Fig. 3, we note that the MH asymmetry is vanishingly small at both the oscillation maxima. If we examine the right panel in Fig. 3, at the first oscillation maximum, one expects large matterinduced changes in probability in comparison to vacuum, which aids the determination of the neutrino mass hierarchy provided the baseline is long enough for matter effects to be substantial.

\section{E. Distinguishing between intrinsic and extrinsic $C P$ contribution}

In the presence of matter, the $C P$ asymmetry can be expressed in terms of intrinsic and extrinsic $C P$ factors [see Eq. (10)]. The issue of separation of the intrinsic contribution from the extrinsic contribution has been addressed in Refs. [48,49], and a useful observable (matter contribution gets approximately canceled) to disentangle the two contributions was suggested. The observable is given by

$$
\delta\left(\Delta P_{\mu e}^{C P}\right)=\Delta P_{\mu e}^{C P}(\delta=\pi / 2)-\Delta P_{\mu e}^{C P}(\delta=0) .
$$

Using this observable, we next depict an oscillogram in the plane of $E$ and $L$ to illustrate separation of the intrinsic versus extrinsic $C P$ contribution in Fig. 4. It is clear that the second oscillation maximum allows for a clean extraction of the intrinsic $C P$ phase. This can be easily explained by Fig. 1, from which we noted that matter influences the probability at the first oscillation maximum far more than it impacts the probability at the location of the second oscillation maximum. This point and the fact that the intrinsic $C P$ asymmetry is larger at the second oscillation

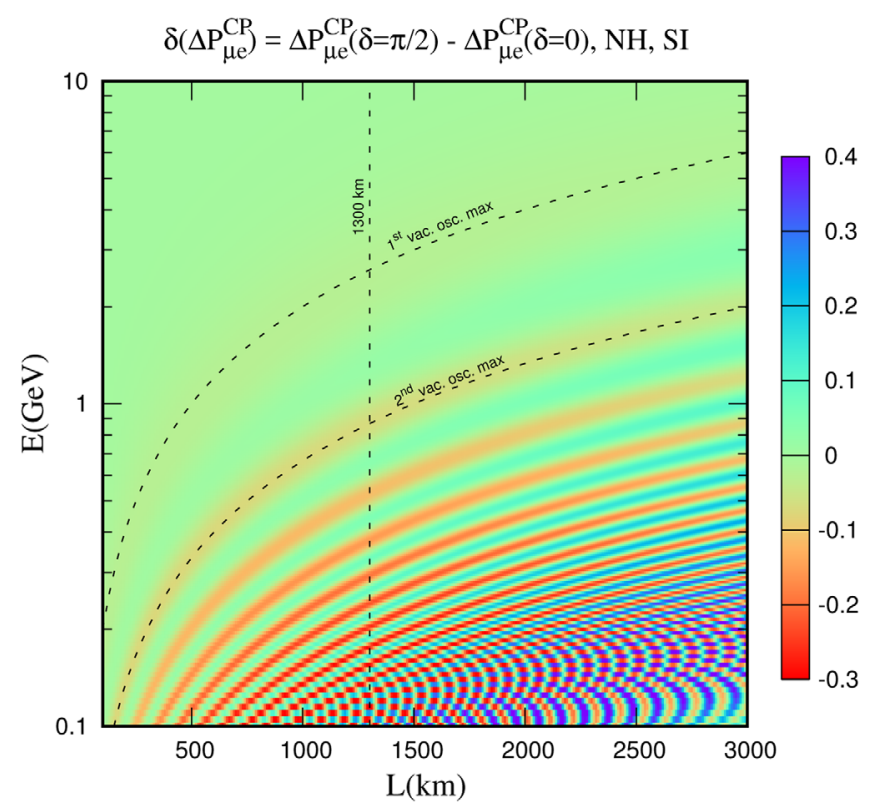

FIG. 4. Oscillogram in the plane of $E$ and $L$ depicting separation of the intrinsic $C P$ contribution from the extrinsic $C P$ contribution. maximum makes the second oscillation maximum the preferred option for addressing the question of separating the intrinsic contribution to the $C P$ phase.

A closer examination of the features in both Figs. 2 and 3 reveals a key phenomenon that is often overlooked in studies of the $C P$ asymmetries in long-baseline oscillations. Both the intrinsic and extrinsic $C P$ asymmetries are largest and changing the most rapidly not at the oscillation maxima but in the region between the maxima-near the oscillation minima. Experimentally, there are few events appearing near oscillation minima, so long-baseline experiments are typically designed to maximize the flux of neutrinos in the region right at the first oscillation maxima-which also occurs at the highest energies and, therefore, highest interaction cross sections and appearance statistics. The optimization of the DUNE experiment specifically targeted optimizing the beam flux to fully cover the region of the first oscillation maxima as well as the region in between $E^{\mathrm{I}}$ and $E^{\mathrm{II}}$ using a genetic algorithm (GA) to optimize the wide-band beam design to maximize the sensitivity to $C P$ violation. This optimized beam, with a decay pipe $195 \mathrm{~m}$ long and $4 \mathrm{~m}$ in diameter, produces a muon neutrino flux that is $20 \%$ greater than the nominal configuration at the first oscillation maximum (between 1.5 and $4 \mathrm{GeV}$ ) and $53 \%$ greater at the second oscillation maximum (between 0.5 and $1.5 \mathrm{GeV}$ ) and reduces the antineutrino contamination of the beam. As shown in the DUNE conceptual design report [8], the GA optimization increased the flux primarily in the region between $E^{\mathrm{I}}$ and $E^{\mathrm{II}}$ and significantly reduced the flux in the region above the $E^{\mathrm{I}}$ oscillation node which contributes to backgrounds below $E^{\mathrm{I}}$. It is for this reason that DUNE can reach the same sensitivities to the three-flavor oscillation parameters using smaller mass detectors compared to similar experiments with flux only in the region around $E^{\mathrm{I}}$.

Our discussion, thus far, has been at the level of probabilities, and, in order to obtain realistic quantitative results, one needs to check the outcome at the level of event rates. The following section covers the experimental details used in our simulations.

\section{EXPERIMENTAL INPUTS}

\section{A. Beam tunes used}

The fluxes considered in the present work are plotted as a function of energy in Fig. 5.

(i) LE flux.-The standard wide-band beam used in our studies of DUNE is derived from a proton beam of energy $80 \mathrm{GeV}[8,50]$ coupled with a target and focusing system design optimized using a GA for maximal sensitivity to $C P$ violation $(\mathrm{CPV})$. By virtue of its wide-band nature, the LE beam is also sensitive to regions above and below the first oscillation maximum and covers part of the region of the second oscillation maximum. In the initial 


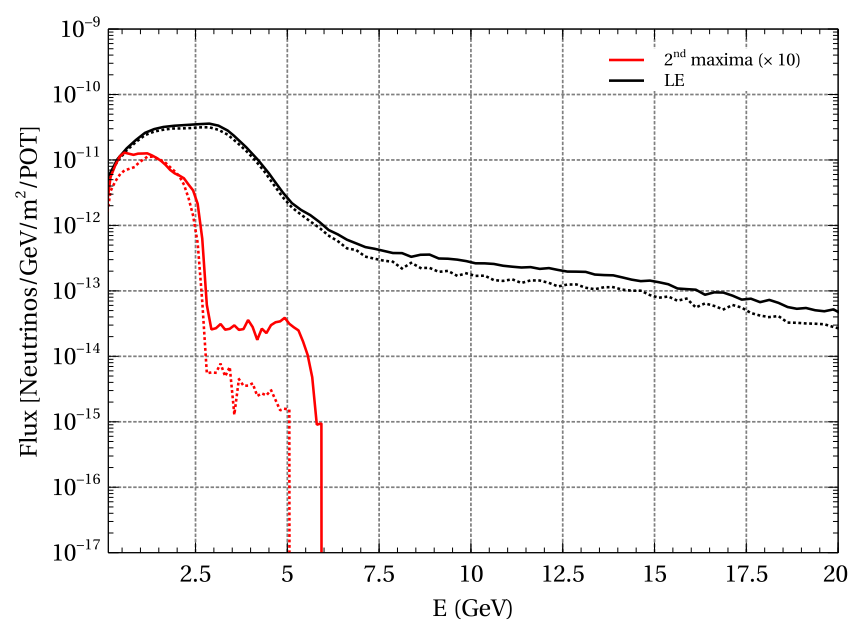

FIG. 5. The fluxes used in the present study. The low energy (LE) flux corresponds to the $C P$ optimized $80 \mathrm{GeV}$ beam [as discussed in the conceptual design report (CDR) [8] ], and the second maxima flux is obtained from a Monte Carlo simulation of an $8 \mathrm{GeV}$ beam using the same target and focusing system design as for the CDR $80 \mathrm{GeV}$ optimized beam. The solid line corresponds to the neutrino mode, while the dotted line corresponds to the antineutrino mode. The fluxes are given in neutrinos per $\mathrm{GeV}$ per $m^{2}$ per proton-on-target (POT). Note that, for equal average beam power, the number of total delivered POTs for the $8 \mathrm{GeV}$ beam will be 10 times larger than that of the $80 \mathrm{GeV}$ beam.

running period of DUNE-here, assumed to be $5 \mathrm{yr}$ - the LE beam will operate at $1.1 \mathrm{MW}$ of power from the Fermilab accelerator complex after the Proton Improvement Plan (PIP) II upgrades. A further set of upgrades aimed at replacing the aging $8 \mathrm{GeV}$ booster (PIP-III) [28] is planned during DUNE operations which will double the power of the LE beam to $2.2 \mathrm{MW}$ at $80 \mathrm{GeV}$.

(ii) Second maxima flux.-The neutrino beam at the second maxima is obtained using a $3 \mathrm{MW}, 8 \mathrm{GeV}$ proton beam which could be generated by the PIP-III SRF linac option [28]. The target and focusing system design is assumed to be identical to the LE beam system. If PIP-III is realized using the pulsed SRF linac option, both the $2.2 \mathrm{MW} 80 \mathrm{GeV}$ wide-band beam and the $8 \mathrm{GeV} 3 \mathrm{MW}$ beam could potentially run simultaneously, which is what is assumed in this study.

The beam line details for the two fluxes are listed in Table II.

\section{B. Detector details}

The DUNE far detector (FD) is described in detail in volume 4 [52] of the DUNE CDR. In our analysis, we have assumed a LAr far detector of fiducial mass $40 \mathrm{kt}$ situated at a distance of $1300 \mathrm{~km}$. We have combined both electron neutrino appearance $\left(\nu_{\mu} \rightarrow \nu_{e}\right)$ and muon neutrino disappearance $\left(\nu_{\mu} \rightarrow \nu_{\mu}\right)$ channels, in both neutrino and antineutrino mode. Details of the experimental configuration and other parameters relevant for DUNE are given in Table III.

\section{EVENT SPECTRUM AT DUNE}

In order to simulate DUNE, we use the GLoBES package $[45,46]$ with the DUNE configuration file provided by the Collaboration [51] as well as for a scenario with improved energy reconstruction capabilities. We implement the density profile of Earth as given by PREM [47]. We obtain our results for the following two scenarios:

(i) the standard case using the available DUNE configuration files [51] with the signal and background smearing matrices obtained from a fast MC (2015 CDR) and

(ii) a case corresponding to improved energy reconstruction capabilities; we implement this by introducing an energy-dependent Gaussian smearing in GLoBES with a resolution of $\Delta E / E=$ $10 \% / \sqrt{E(\mathrm{GeV})}$ and $15 \% / \sqrt{E(\mathrm{GeV})}$, respectively, for the $\nu_{e} \mathrm{CC}$ and $\nu_{\mu} \mathrm{CC}$ signal and backgrounds. The $\mathrm{NC}$ and $\nu_{\tau}$ backgrounds remain the same as in (i). We note that, in the latest studies of DUNE performance reported in the 2020 technical design report, the average energy resolution of $\nu_{e}$ and $\nu_{\mu}$ CC events obtained using full simulation and reconstruction is $13 \%$ and $18 \%$, respectively [53]. It is possible that

TABLE II. Beam line parameters assumed for the different design fluxes used in our sensitivity calculations [7,11,51]. The LBNF neutrino beam line decay pipe length has been chosen to be $194 \mathrm{~m}$.

\begin{tabular}{llc}
\hline \hline Parameter & \multicolumn{1}{c}{ LE (CPV-optimized design) } & Second maxima \\
\hline Proton beam energy & $80 \mathrm{GeV}$ & $8 \mathrm{GeV}$ \\
Proton beam power & $1.1 \mathrm{MW}($ PIP-II) $/ 2.2 \mathrm{MW}$ (PIP-III) & $3 \mathrm{MW}(\mathrm{PIP}-\mathrm{III})$ \\
POT per year & $1.47 \times 10^{21} / 2.94 \times 10^{21}$ & $40.1 \times 10^{21}$ \\
Focusing & Two horns, GA optimized for CPV sensitivity $(2015)$ & $\sim 300 \mathrm{kA}$ \\
Horn current & $\sim 300 \mathrm{kA}$ & $200 \mathrm{~m}$ \\
Decay pipe length & $194 \mathrm{~m}$ & $4 \mathrm{~m}$ \\
Decay pipe diameter & $4 \mathrm{~m}$ & \\
& $\mathrm{GA} \rightarrow$ genetic algorithm & \\
\hline \hline
\end{tabular}


TABLE III. Details of the experimental configuration and other parameters relevant for DUNE.

\begin{tabular}{lll}
\hline \hline S. No. & Relevant parameter & \\
\hline 1 & Location & DSA \\
2 & Status & Under construction \\
3 & Accelerator facility & Fermilab \\
4 & Beam power & $1.1 \mathrm{MW}$ at $80 \mathrm{GeV}$ (PIP-II), 2.2 MW at $80 \mathrm{GeV}$ (PIP-III) \\
& & $3 \mathrm{MW}$ at $8 \mathrm{GeV}$ (PIP-III SRF linac option) \\
5 & Expected POT/yr & See Table II \\
6 & Baseline length & $1300 \mathrm{~km}$ \\
7 & Off-axis angle & 0 \\
8 & Detector technology & LAr \\
9 & Fiducial mass & $40 \mathrm{kt}$ \\
10 & Run times & $5 \mathrm{yr}$ with $80 \mathrm{GeV}$ at $1.1 \mathrm{MW}$ \\
& & $+5 \mathrm{yr}$ with $80 \mathrm{GeV}$ at $2.2 \mathrm{MW}$ \\
& & $+5 \mathrm{yr}$ with $8 \mathrm{GeV}$ at $3 \mathrm{MW}$ \\
11 & & {$[0.125,18.0]$} \\
12 & Energy window $(\mathrm{GeV})$ & 71 \\
14 & Energy bins & Migration matrices based on fast MC (2015 CDR) \\
& Energy resolution, $\sigma_{e}$ & $\nu_{e}: 2 \%$ (signal) $\nu_{e}: 5 \%$ (bkgd) \\
\hline \hline
\end{tabular}

TABLE IV. Total number of event rates for different beam tune combinations [the signal events for combination (c) when improved energy reconstruction is taken into account are given in parentheses].

\begin{tabular}{|c|c|c|c|c|c|c|}
\hline \multirow[b]{2}{*}{ Beam options } & \multicolumn{2}{|c|}{$\nu_{\mu} \rightarrow \nu_{e}$} & \multicolumn{2}{|c|}{$\bar{\nu}_{\mu} \rightarrow \bar{\nu}_{e}$} & \multirow{2}{*}{$\frac{\nu_{\mu} \rightarrow \nu_{\mu}}{\mathrm{NH}}$} & \multirow{2}{*}{$\frac{\bar{\nu}_{\mu} \rightarrow \bar{\nu}_{\mu}}{\mathrm{NH}}$} \\
\hline & $\mathrm{NH}$ & $\mathrm{IH}$ & NH & $\mathrm{IH}$ & & \\
\hline \multicolumn{7}{|c|}{ (a) LE, $1.1 \mathrm{MW}+\mathrm{LE}, 2.2 \mathrm{MW}$} \\
\hline Signal $\delta=0$ & 3222 & 1759 & 859 & 1303 & 13415 & 6158 \\
\hline Signal $\delta=\pi / 2$ & 2727 & 1481 & 928 & 1463 & & \\
\hline Signal $\delta=-\pi / 2$ & 3784 & 2167 & 742 & 1130 & & \\
\hline $\operatorname{Bkgd}\left(\bar{\nu}_{e}+\nu_{e}\right) \mathrm{CC}$ & 446 & 461 & 227 & 224 & & \\
\hline $\operatorname{Bkgd}\left(\bar{\nu}_{\mu}+\nu_{\mu}\right) \mathrm{CC}$ & 6 & 6 & 4 & 3 & & \\
\hline $\operatorname{Bkgd}\left(\bar{\nu}_{\tau}^{\mu}+\nu_{\tau}\right) \mathrm{CC}$ & 43 & 44 & 25 & 25 & 61 & 38 \\
\hline Bkgd NC & 55 & 55 & 27 & 27 & 155 & 81 \\
\hline $\operatorname{Bkgd} \nu_{\mu} \mathrm{CC}$ & & & & & & 1536 \\
\hline $\operatorname{Bkgd} \bar{\nu}_{\mu} \mathrm{CC}$ & & & & & 547 & \\
\hline \multicolumn{7}{|c|}{ (b) Second maxima, $3 \mathrm{MW}$} \\
\hline Signal $\delta=0$ & 208 & 121 & 40 & 82 & 1902 & 663 \\
\hline Signal $\delta=\pi / 2$ & 178 & 93 & 45 & 85 & & \\
\hline Signal $\delta=-\pi / 2$ & 292 & 175 & 30 & 58 & & \\
\hline $\operatorname{Bkgd}\left(\bar{\nu}_{e}+\nu_{e}\right) \mathrm{CC}$ & 37 & 38 & 13 & 12 & & \\
\hline $\operatorname{Bkgd}\left(\bar{\nu}_{\mu}+\nu_{\mu}\right) \mathrm{CC}$ & 1 & 1 & 1 & 1 & & \\
\hline $\operatorname{Bkgd}\left(\bar{\nu}_{\tau}+\nu_{\tau}\right) \mathrm{CC}$ & 0 & 0 & 0 & 0 & 0 & 0 \\
\hline Bkgd NC & 4 & 4 & 1 & 1 & 11 & 5 \\
\hline $\operatorname{Bkgd} \nu_{\mu} \mathrm{CC}$ & & & & & & 27 \\
\hline $\operatorname{Bkgd} \bar{\nu}_{\mu} \mathrm{CC}$ & & & & & 8 & \\
\hline \multicolumn{7}{|c|}{ (c) LE, 1.1 MW + LE, $2.2 \mathrm{MW}+$ second maxima, $3 \mathrm{MW}$} \\
\hline Signal $\delta=0$ & $3430(3455)$ & $1880(1876)$ & $899(883)$ & $1384(1377)$ & $15317(15707)$ & $6821(6722)$ \\
\hline Signal $\delta=\pi / 2$ & $2904(2922)$ & $1574(1561)$ & $973(958)$ & $1548(1541)$ & & \\
\hline Signal $\delta=-\pi / 2$ & $4076(4123)$ & $2342(2348)$ & $772(754)$ & $1188(1176)$ & & \\
\hline $\operatorname{Bkgd}\left(\bar{\nu}_{e}+\nu_{e}\right) \mathrm{CC}$ & 483 & 499 & 239 & 236 & & \\
\hline $\operatorname{Bkgd}\left(\bar{\nu}_{\mu}+\nu_{\mu}\right) \mathrm{CC}$ & 7 & 7 & 4 & 4 & & \\
\hline $\operatorname{Bkgd}\left(\bar{\nu}_{\tau}+\nu_{\tau}\right) \mathrm{CC}$ & 43 & 44 & 25 & 25 & 61 & 38 \\
\hline Bkgd NC & 59 & 59 & 28 & 28 & 166 & 86 \\
\hline $\operatorname{Bkgd} \nu_{\mu} \mathrm{CC}$ & & & & & & 1563 \\
\hline $\operatorname{Bkgd} \bar{\nu}_{\mu} \mathrm{CC}$ & & & & & 555 & \\
\hline
\end{tabular}


TABLE V. Number of $\nu_{\mu} \rightarrow \nu_{e} \mathrm{CC}$ events and total background in the region of the secondary oscillation nodes $(0.125-1.5 \mathrm{GeV}$ reconstructed energy) from the PIP-III $80 \mathrm{GeV} 2.2 \mathrm{MW}$ and $8 \mathrm{GeV} 3 \mathrm{MW}$ beams (we have listed the events for improved energy reconstruction in parentheses).

\begin{tabular}{lrr}
\hline \hline & \multicolumn{2}{c}{$\nu_{\mu} \rightarrow \nu_{e}$} \\
\cline { 2 - 3 } Beam options & $\mathrm{NH}$ & \multicolumn{1}{c}{$\mathrm{IH}$} \\
\hline (a) LE, $2.2 \mathrm{MW}$ & $373(134)$ & $219(111)$ \\
Signal $\delta=0$ & $333(115)$ & $175(105)$ \\
Signal $\delta=\pi / 2$ & $508(181)$ & $290(169)$ \\
Signal $\delta=-\pi / 2$ & $88(65)$ & $90(65)$ \\
Bkgd & & $77(58)$ \\
(b) Second maxima, 3 MW & $120(75)$ & $62(51)$ \\
Signal $\delta=0$ & $100(55)$ & $125(107)$ \\
Signal $\delta=\pi / 2$ & $177(110)$ & $28(21)$ \\
Signal $\delta=-\pi / 2$ & $27(21)$ & \\
Bkgd & \multicolumn{2}{c}{} \\
\hline \hline
\end{tabular}

further improvements in reconstruction algorithms could reach the performance we assumed above.

We consider the following beam and runtime combinations: (a) LE, 1.1 MW + LE, 2.2 MW $\rightarrow$ run time of $5 \mathrm{yr}$ in LE, 1.1 MW and $5 \mathrm{yr}$ in LE, 2.2 MW distributed equally in neutrino and antineutrino modes;

(b) second maxima, $3 \mathrm{MW} \rightarrow$ run time of $5 \mathrm{yr}$ distributed equally in neutrino and antineutrino modes;

(c) LE, $1.1 \mathrm{MW}+\mathrm{LE}, \quad 2.2 \mathrm{MW}+$ second maxima, $3 \mathrm{MW} \rightarrow$ run time of $5 \mathrm{yr}$ in LE, $1.1 \mathrm{MW}, 5 \mathrm{yr}$ in LE, $2.2 \mathrm{MW}$ and $5 \mathrm{yr}$ with second maxima, $3 \mathrm{MW}$ distributed equally in neutrino and antineutrino modes. It is assumed the $8 \mathrm{GeV} 3 \mathrm{MW}$ beam option (second maxima) runs concurrently with the $80 \mathrm{GeV} 2.2 \mathrm{MW}$ (LE) default beam using the same focusing system.

Table IV lists the total signal and background events for the three beam combinations mentioned above for the $\nu_{e}$ appearance and $\nu_{\mu}$ disappearance channels. In Table V, we list the number of signal and total background events in the region of the secondary oscillation nodes (second maxima region and below) in the two beam options from the Fermilab PIP-III upgrade-the $2.2 \mathrm{MW} 80 \mathrm{GeV}$ beam and the $3 \mathrm{MW} 8 \mathrm{GeV}$ beam. The addition of running with the $8 \mathrm{GeV} 3 \mathrm{MW}$ enhances the default DUNE event yield at the secondary oscillation nodes by $\sim 50 \%-60 \%$.

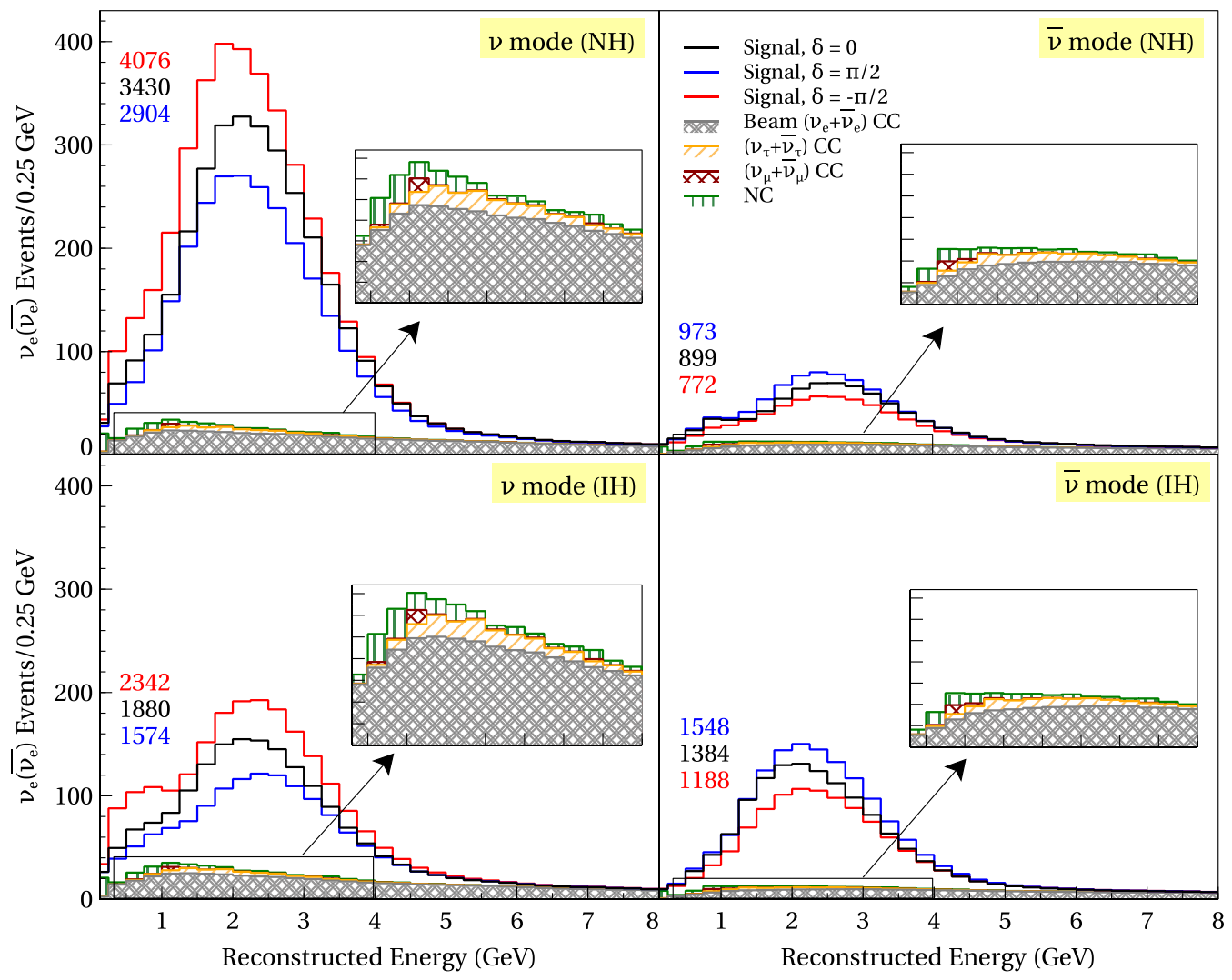

FIG. 6. Electron (anti)neutrino appearance event spectrum with a $40 \mathrm{kt}$ DUNE far detector using DUNE configuration files [51] for the beam combination (c): LE 1.1 MW beam, LE 2.2 MW beam, and second maxima $3 \mathrm{MW}$ beam for different values of $\delta$ $(\delta=0, \pi / 2,-\pi / 2)$. The run time of $15 \mathrm{yr}$ is split equally among the three beams ( $5 \mathrm{yr}$ each) and distributed evenly between neutrino and antineutrino modes $(2.5 \mathrm{yr}+2.5 \mathrm{yr})$ for each beam tune. The event rates are stacked from below backgrounds. The total number of events corresponding to a particular value of $\delta$ is mentioned in each panel. 
The event spectrum for option (c) is shown in Figs. 6-9. Figures 6 and 7 correspond to $\nu_{e}$ appearance events, while Figs. 8 and 9 show the $\nu_{\mu}$ disappearance events for the two scenarios. In general, one notes that the events are peaked at the value of energy where the flux is largest-for the $80 \mathrm{GeV} \mathrm{LE}$ beam, the events peak around $2-3 \mathrm{GeV}$, while for the $8 \mathrm{GeV}$ beam, it is around $0.8-0.9 \mathrm{GeV}$. The $\delta$ dependence of the event spectrum can be understood from the $\delta$ dependence of the probabilities.

The signal for $\nu_{e}$ appearance is an excess of chargedcurrent (CC) $\nu_{e}$ and $\bar{\nu}_{e}$ interactions over the expected background in the far detector. The background to $\nu_{e}$ appearance is composed of

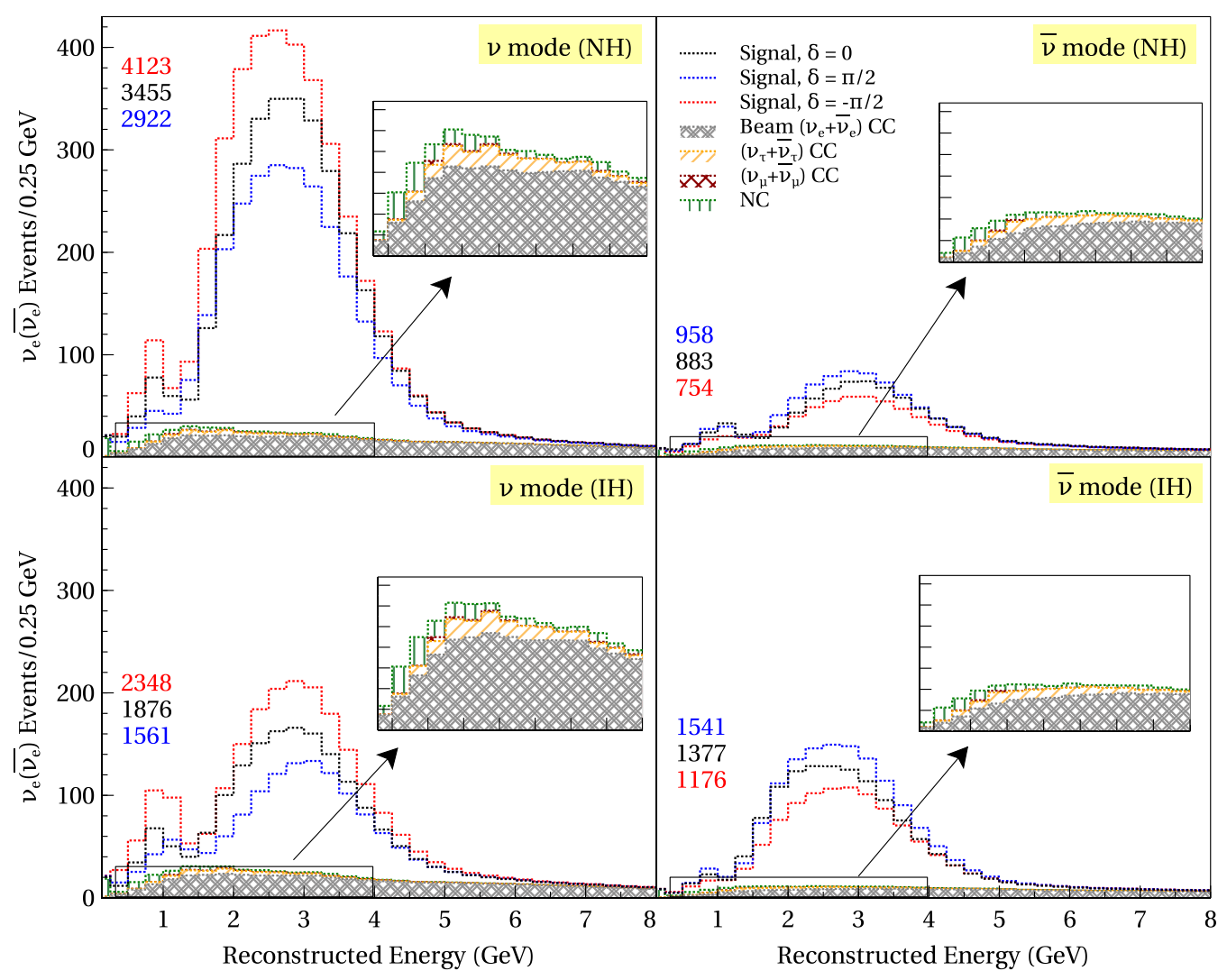

FIG. 7. The same as Fig. 6 with improved energy reconstruction capabilities shown using dotted lines.

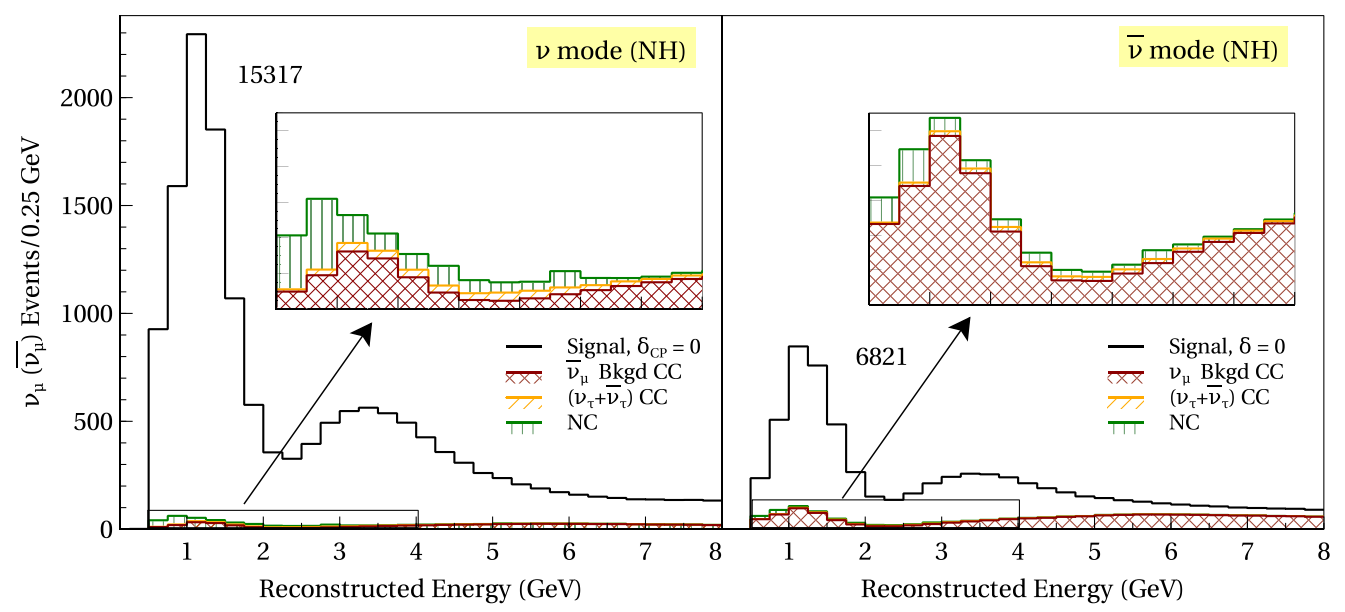

FIG. 8. Muon (anti)neutrino disappearance event spectrum with a $40 \mathrm{kt}$ DUNE far detector for the combination (c): LE $1.1 \mathrm{MW}$ beam, LE 2.2 MW beam, and second maxima $3 \mathrm{MW}$ beam for $\delta=0$. The run time is split equally among the three beams (5 yr each) and distributed evenly between neutrino and antineutrino modes $(2.5 \mathrm{yr}+2.5 \mathrm{yr})$. 


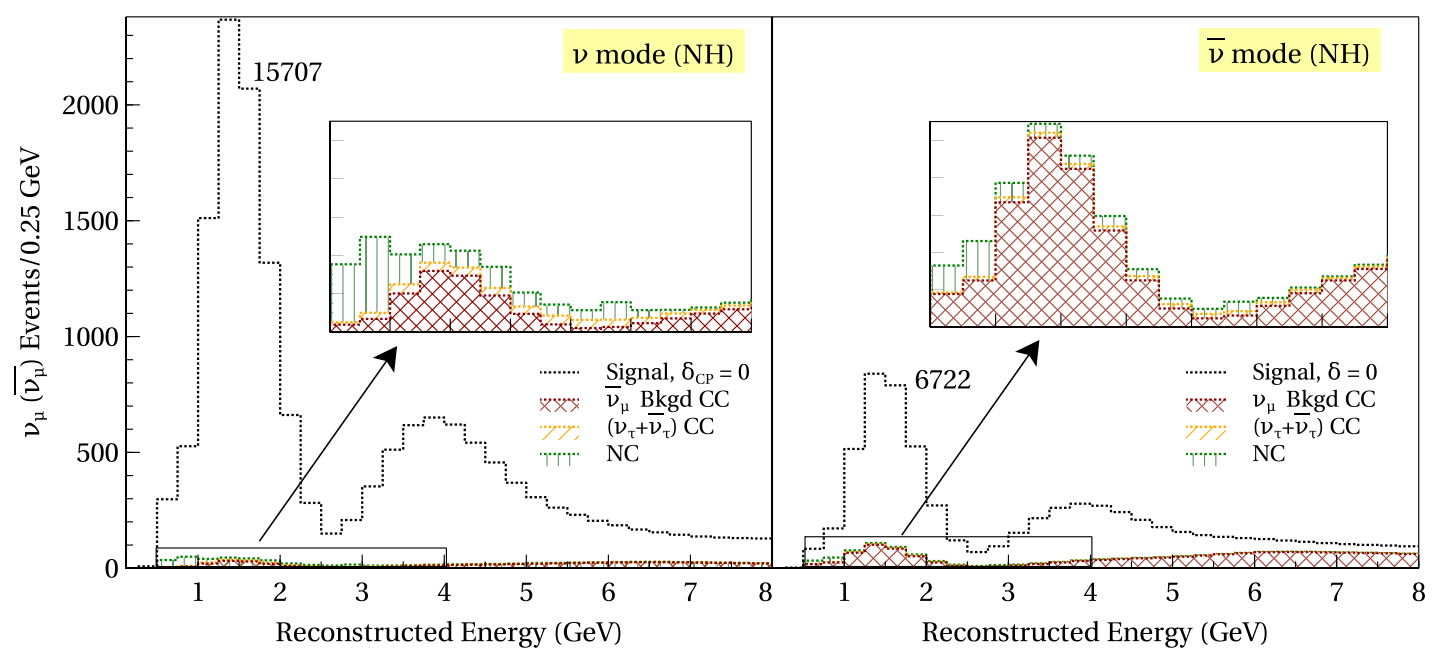

FIG. 9. The same as Fig. 8 with improved energy reconstruction capabilities shown using dotted lines.

(i) beam $\left(\nu_{e}+\bar{\nu}_{e}\right)$ CC. $-\mathrm{CC}$ interactions of $\nu_{e}$ and $\bar{\nu}_{e}$ intrinsic to the beam;

(ii) $\left(\nu_{\tau}+\bar{\nu}_{\tau}\right)$ CC. $-\nu_{\tau}$ and $\bar{\nu}_{\tau}$ CC events in which the $\tau$ 's decay leptonically into electrons or positrons.

(iii) $\left(\nu_{\mu}+\bar{\nu}_{\mu}\right)$ CC. - misidentified $\nu_{\mu}$ and $\bar{\nu}_{\mu}$ CC events;

(iv) NC.- - neutral-current backgrounds.

It should be noted that, though $\mathrm{NC}$ and $\nu_{\tau}$ backgrounds are due to interactions of higher-energy neutrinos, they contribute to backgrounds mainly at lower energies, which impacts sensitivity to $C P$ violation.

\section{ANALYSIS PROCEDURE}

To estimate the sensitivities of DUNE to $C P$ violation, $\mathrm{MH}$, and the octant of $\theta_{23}$, we perform a standard $\chi^{2}$ analysis. Even though all results are produced numerically with the help of the GLoBES software, in order to gain insight, let us examine the analytical form of the $\chi^{2}$ relevant for each of the mentioned unknowns.

\section{A. Sensitivity to $C P$ violation}

Including only statistical effects, the $\chi^{2}$ for $C P$-violation sensitivity for a given oscillation channel (say, $\nu_{\mu} \rightarrow \nu_{e}$ ) is given by $[40,54]$

$\chi^{2} \equiv \min _{\delta_{\text {test }}} \sum_{i=1}^{x} \sum_{j=\nu, \bar{\nu}} \frac{\left[N_{\text {true }}^{i, j}\left(\delta_{\text {true }}\right)-N_{\text {test }}^{i, j}\left(\delta_{\text {test }}=0, \pi\right)\right]^{2}}{N_{\text {true }}^{i, j}\left(\delta_{\text {true }}\right)}$,

where $N_{\text {true }}^{i, j}$ and $N_{\text {test }}^{i, j}$ are the number of true and test events in the $\{i, j\}$ th bin, respectively. ${ }^{2}$ The index $i$ corresponds to energy bins $(i=1 \rightarrow x)$, and the number of bins depends

\footnotetext{
${ }^{2} N_{\sigma}=\sqrt{\Delta \chi^{2}} \cdot \Delta \chi^{2}=\chi^{2}$ as we have not included any fluctuations in simulated data. This is Pearson's definition of $\chi^{2}$ [55]. For a large sample size, the other definition using log-likelihood also yields similar results.
}

upon the particular experiment under consideration. For the case of DUNE, there are 64 bins of width $125 \mathrm{MeV}$ in $0-8 \mathrm{GeV}$ and seven unequal bins in $8-20 \mathrm{GeV}$. $j$ is being summed over the neutrino and antineutrino contribution. In order to determine the $\chi^{2}$ that concerns the sensitivity to $C P$ violation, the test value of phase $(\delta)$ is assumed to be 0 or $\pi$ and the $\chi^{2}$ for any true value of phase $(\delta)$ in the full range of $[-\pi, \pi]$ is computed.

The characteristic double peak shape of the curves is expected, since the sensitivity drops to zero at the $C P$-conserving values while it is maximum at the maximal $C P$-violating values $(\delta= \pm \pi / 2)$. The $\chi^{2}$ is computed as given in Eq. (15) for a given set of true values by minimizing over the test parameters, and this procedure is repeated for all possible true values listed in Table I. Some general remarks relevant for computation of $\chi^{2}$ for this and other unknowns are in order. We have marginalized over the standard oscillation parameters. It should be noted that the total $\chi^{2}$ is a sum of contributions from the two channels $\left(\nu_{\mu} \rightarrow \nu_{e}\right.$ and $\left.\nu_{\mu} \rightarrow \nu_{\mu}\right)$.

\section{B. Sensitivity to the MH}

The determination of the $\mathrm{MH}$ is a measurement of a binary value, namely, the sign of the difference of the square of the masses of the 1 and 3 states. Therefore, there can be only two possibilities for the true choice of hierarchy ( $\mathrm{NH}$ or $\mathrm{IH}$ ), and one would like to decipher the sensitivity of DUNE to figure out the correct MH.

In order to understand the features of the sensitivity plots (considering the true hierarchy as $\mathrm{NH}$ or $\mathrm{IH}$ ), we give a statistical definition of $\chi^{2}$ as follows [56]:

$$
\chi^{2} \equiv \min _{\delta_{\text {test }}} \sum_{i=1}^{x} \sum_{j=\nu, \bar{\nu}} \frac{\left[N_{\mathrm{NH}}^{i, j}\left(\delta_{\text {true }}\right)-N_{\mathrm{IH}}^{i, j}\left(\delta_{\text {test }}\right)\right]^{2}}{N_{\mathrm{NH}}^{i, j}\left(\delta_{\text {true }}\right)},
$$


where the true hierarchy is taken to be $\mathrm{NH} . N_{\mathrm{NH}}^{i, j}$ and $N_{\mathrm{IH}}^{i, j}$ are the number of $\mathrm{NH}$ and $\mathrm{IH}$ events in the $\{i, j\}$ th bin, respectively. The index $i$ corresponds to energy bins $(i=1 \rightarrow x)$, and the number of bins depends upon the particular experiment under consideration. For the case of DUNE, there are 64 bins of width $125 \mathrm{MeV}$ in $0-8 \mathrm{GeV}$ and seven unequal bins in $8-20 \mathrm{GeV}$. $j$ is being summed over the neutrino and antineutrino contribution.

The $\chi^{2}$ is computed as given in Eq. (16) for a given set of true values by minimizing over the test parameters, and this procedure is repeated for all possible true values listed in Table I. The shape of the sensitivity curves can be explained using the analytic form of probabilities [54,56].

\section{Sensitivity to the octant of $\boldsymbol{\theta}_{23}$}

It is important to determine the value of $\sin ^{2} \theta_{23}$ with sufficient precision to determine the octant of $\theta_{23}$. A combination of $\nu_{e}$ appearance (which is sensitive to $\sin ^{2} \theta_{23}$ ) and $\nu_{\mu}$ disappearance (sensitive to $\sin ^{2} 2 \theta_{23}$ ) measurements would allow us to probe both maximal mixing and the octant of $\theta_{23}$. The $\Delta \chi^{2}$ is defined as [8]

$$
\Delta \chi_{\text {octant }}^{2}=\left|\chi_{\theta_{23}^{\text {test }}>45^{\circ}}^{2}-\chi_{\theta_{23}^{\text {test }}<45^{\circ}}^{\text {e }}\right|
$$

where the value of $\theta_{23}$ in the wrong octant is constrained only to have a value within the wrong octant (i.e., it is not required to have the same value of $\sin ^{2} 2 \theta_{23}$ as the true value).

\section{RESULTS}

One of the primary objectives of DUNE is to ascertain whether $C P$ is violated in the leptonic sector within the context of the standard three-flavor mixing scenario. In Fig. 10, we depict the $C P$-violation sensitivity as a function of $\delta$ for different beam tune combinations. The largest sensitivity to $C P$ violation occurs at $\delta= \pm \pi / 2$. This is expected, because the difference between the event rates at $\delta= \pm \pi / 2$ (maximal $C P$-violating values) and $\delta=0$ or $\pi$ (CP-conserving values) is the largest. The contribution from the second maxima beam (brown curve) alone stays below $3 \sigma$ level for all values of the $C P$ phase even with the improved energy resolution. The contribution from LE 1.1 MW and LE 2.2 MW beam taken together is shown as a black curve, and, for the considered exposure $(5+5 \mathrm{yr})$, the sensitivity lies above $3 \sigma$ level for $\sim 74 \%(78 \%)$ of the possible values of $\delta$ for $\mathrm{NH}(\mathrm{IH})$. The combination LE 1.1 MW and LE 2.2 MW beam along with second maxima beam (running concurrently with LE $2.2 \mathrm{MW}$ ) produces a modest improvement in the sensitivity to $C P$ violation near the maximal $C P$-violating values. This holds irrespective of the choice of hierarchy. We depict the same information in Fig. 11 but as a function of the fraction of values of $\delta$ that allow for discovery of $C P$ violation at the corresponding significance. We note that improved energy reconstruction capabilities with better neutrino energy resolution could lead to much better significance (as shown by the dashed lines) especially in the neighborhood of maximal $C P$-violating values.

In Fig. 12, we show sensitivity to the $\mathrm{MH}$ as a function of $\delta$. Here again, we get significantly better results for the

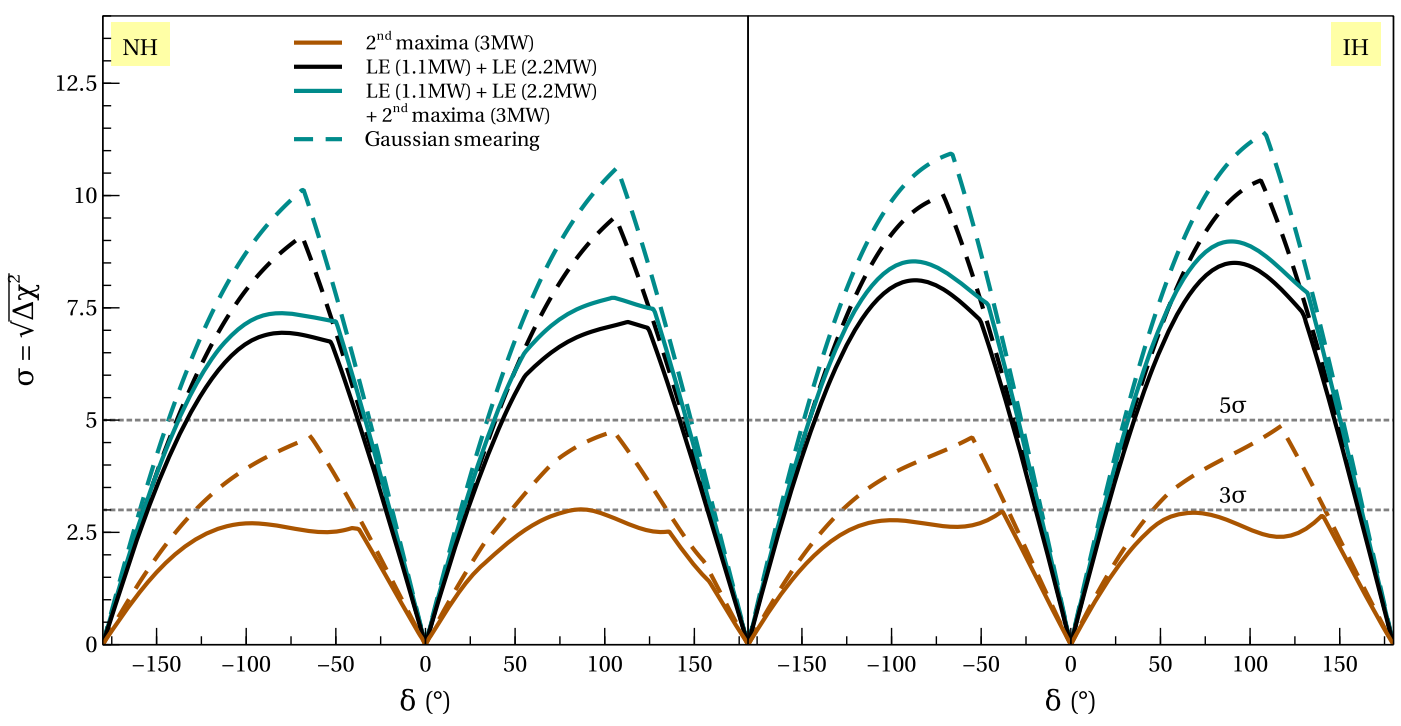

FIG. 10. Sensitivity to $C P$ violation as a function of $\delta$. The three curves correspond to (a) second maxima $3 \mathrm{MW}$ beam (2.5 yr $\nu+$ $2.5 \mathrm{yr} \bar{\nu})$, (b) LE 1.1 MW beam (2.5 yr $+2.5 \mathrm{yr} \bar{\nu})+\mathrm{LE} 2.2 \mathrm{MW}$ beam $(2.5 \mathrm{yr} \nu+2.5 \mathrm{yr} \bar{\nu})$, and (c) LE $1.1 \mathrm{MW}$ beam $(2.5 \mathrm{yr} \nu+$ $2.5 \mathrm{yr} \bar{\nu})+$ LE 2.2 MW beam $(2.5 \mathrm{yr} \nu+2.5 \mathrm{yr} \bar{\nu})+$ second maxima $3 \mathrm{MW}$ beam $(2.5 \mathrm{yr} \nu+2.5 \mathrm{yr} \bar{\nu})$. The solid lines (in this and the following figures) depict the standard case with the DUNE configuration files [51], while the dashed lines depict the scenario with improved neutrino energy resolution (Gaussian smearing). 


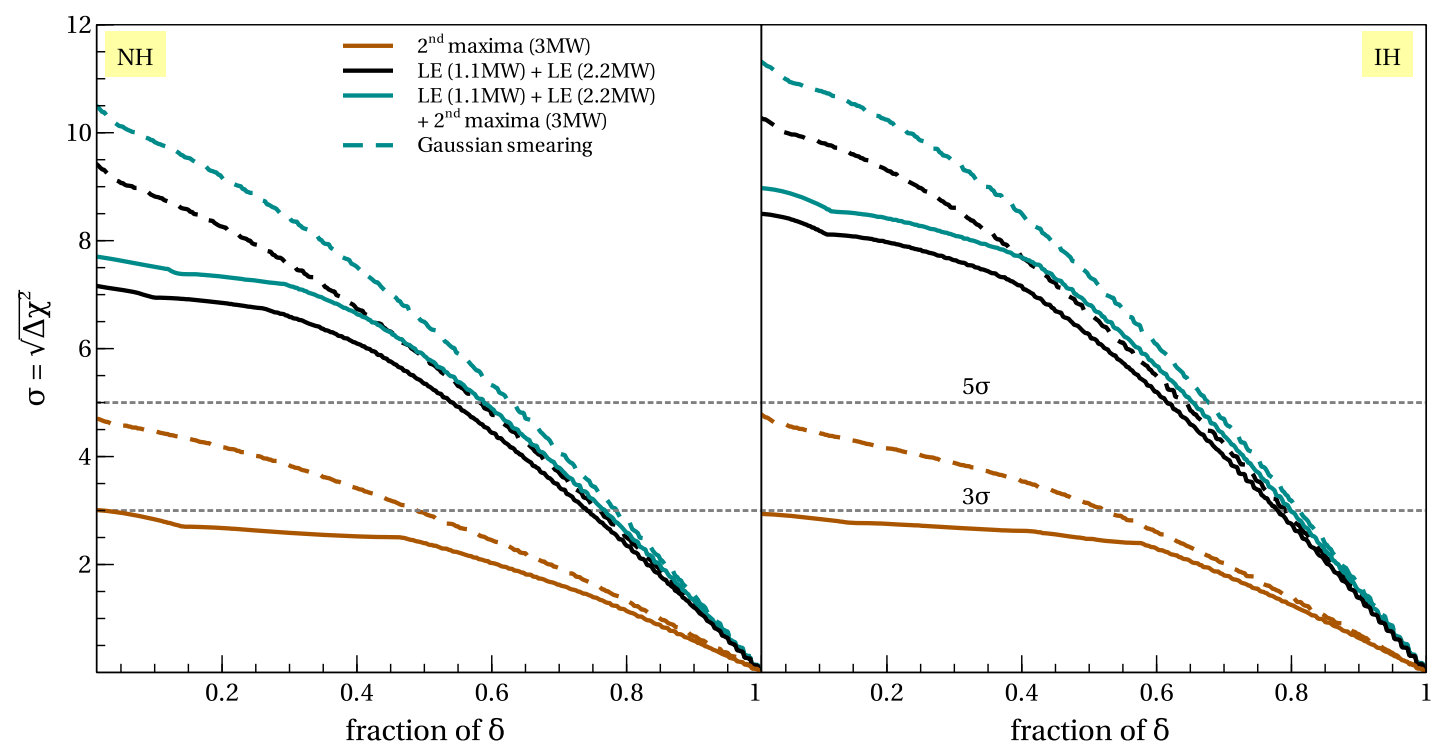

FIG. 11. Sensitivity to $C P$ violation as a function of the fraction of values of $\delta$ for which a given significance could be achieved for different beam combinations and with improved neutrino energy resolution (Gaussian smearing).

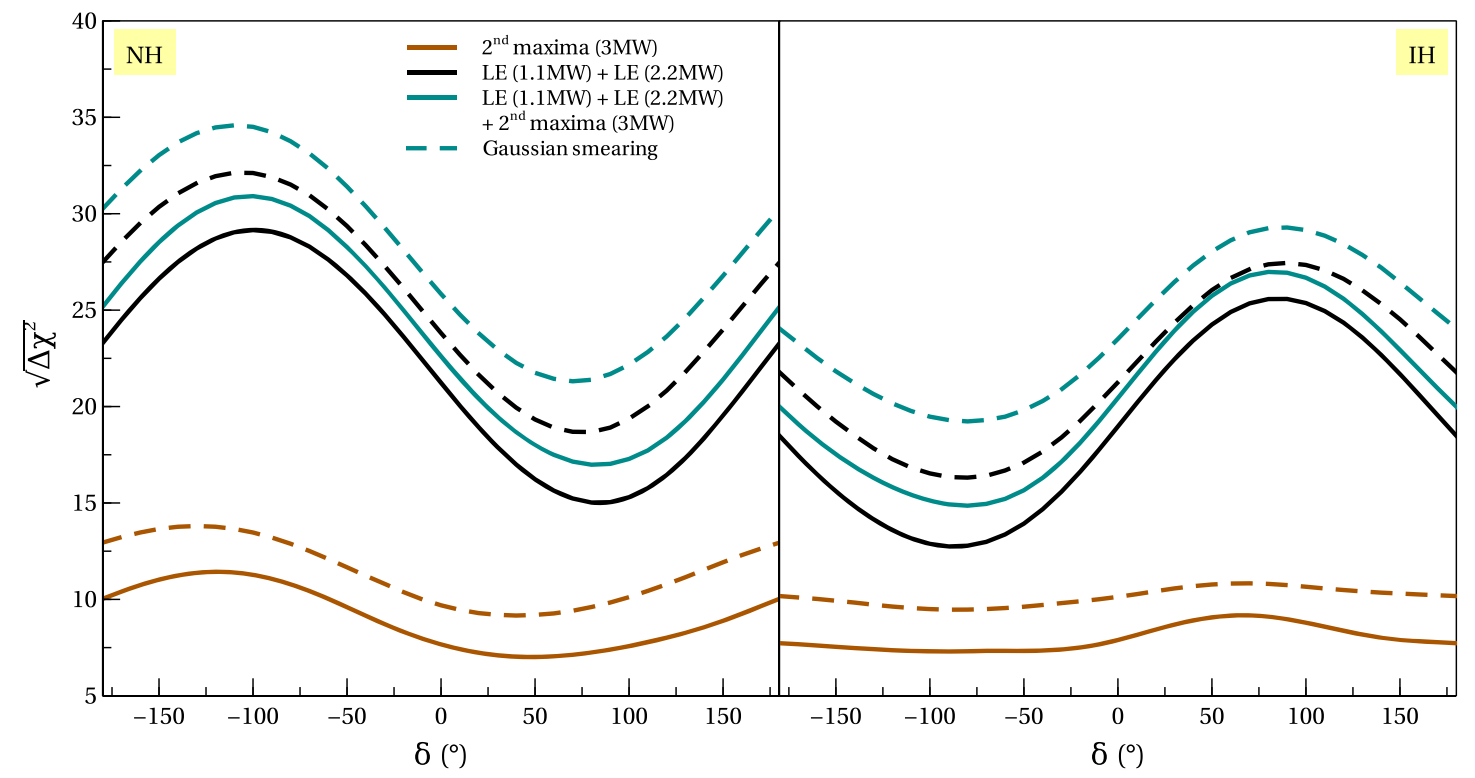

FIG. 12. Sensitivity to the neutrino mass hierarchy as a function of $\delta$ for various beam combinations and with improved neutrino energy resolution (Gaussian smearing).

LE beam running only as compared to the second maxima beam, when taken in isolation. However, when we consider the combination of beam tunes (LE 1.1 MW, LE 2.2 MW, and second maxima), we notice a modest overall improvement in the sensitivity to $M H$ for all values of $\delta$. With the exposure considered, one can discern the $\mathrm{MH}$ definitively ${ }^{3}$ for the LE beam combination as well as for the combination of LE with the second maxima. With better energy

\footnotetext{
${ }^{3}$ See $[55,57]$ for a discussion on statistical methods in estimation of the neutrino $\mathrm{MH}$.
}

reconstruction capabilities, we find that the $\mathrm{MH}$ can be deciphered even better for all values of $\delta$.

DUNE will not only address questions pertaining to $C P$ violation and neutrino $\mathrm{MH}$, but also improve the precision on key parameters $\left(\sin ^{2} \theta_{23}\right.$ and the octant of $\theta_{23}, \delta$, $\sin ^{2} 2 \theta_{13}$, and $\Delta m_{31}^{2}$ ) entering the oscillation framework. It is crucial to determine the value of $\sin ^{2} \theta_{23}$ with sufficient precision to determine the octant. The sensitivity of determining the octant of $\theta_{23}$ as a function of the true value of $\theta_{23}$ for different beam combinations is shown in Fig. 13 for $\mathrm{NH}$ and $\mathrm{IH}$. Adding the $8 \mathrm{GeV}$ beam data to 


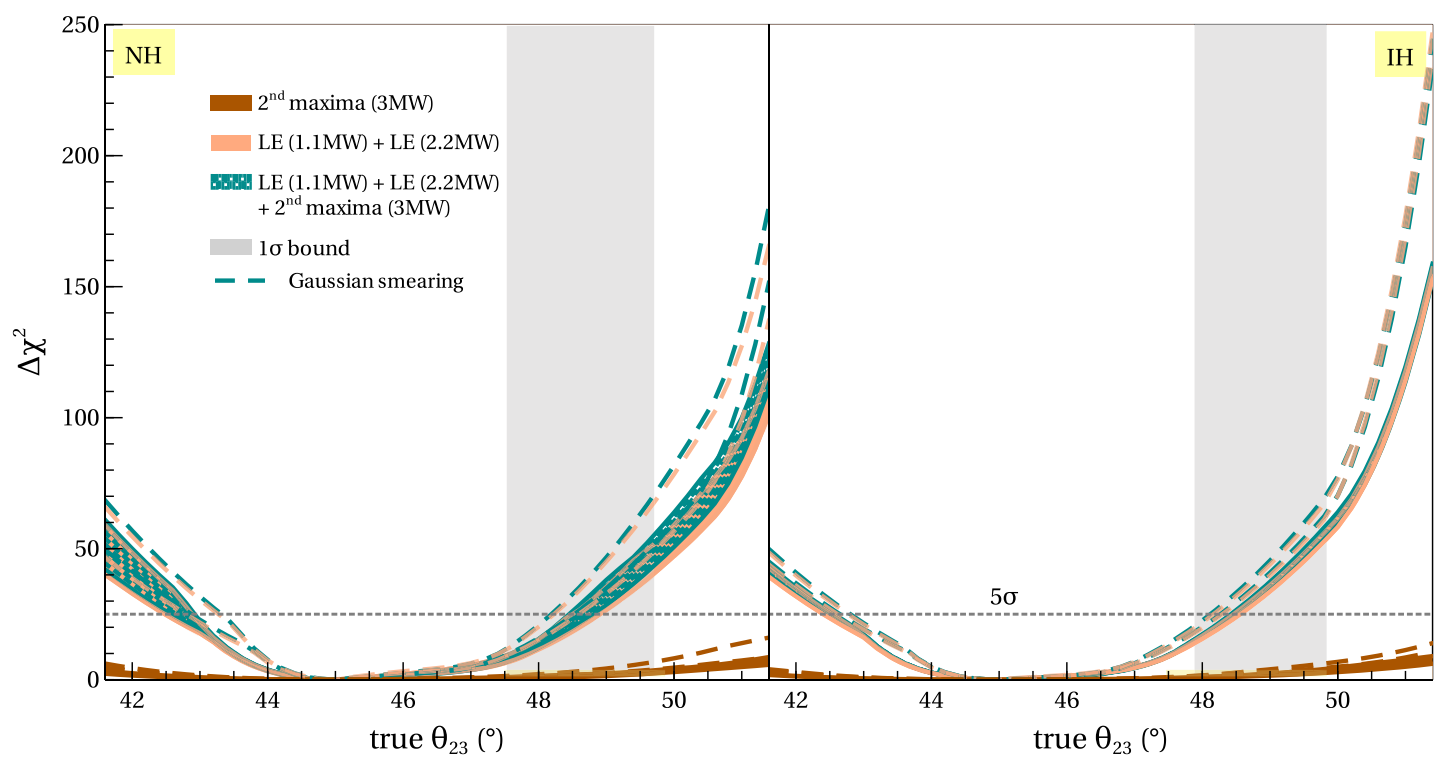

FIG. 13. The significance with which DUNE can resolve the $\theta_{23}$ octant as a function of the true value of $\theta_{23}$ for different combinations of beams and with improved neutrino energy resolution (Gaussian smearing). The shaded band around the curve represents the range in sensitivity due to potential variations in the true value of $\delta$. The gray shaded regions indicate the current $1 \sigma$ bounds on the value of $\theta_{23}$ from a global fit.

$80 \mathrm{GeV}$ data at DUNE improves the sensitivity to resolution of the $\theta_{23}$ octant degeneracy. The width of each curve is due to the unknown $C P$ phase and covers all possible true $\delta$ values. The gray shaded regions indicate the current $1 \sigma$ bounds on the value of $\theta_{23}$ from a global fit [3]. In this fit, muon neutrino disappearance contributes to the precision on $\sin ^{2} 2 \theta_{23}$, while the electron neutrino appearance data provides information on the $\theta_{23}$ octant. A $5 \sigma$ determination of the octant of $\theta_{23}$ will be possible for at least $90 \%$ of true values of $\delta$ for $42.5^{\circ}<\theta_{23}<49^{\circ}$ for $\mathrm{NH}$. Most of the sensitivity comes from the LE data, because the second maximum does not provide any added sensitivity to the octant determination. We show the impact of improved energy reconstruction capabilities on the octant of $\theta_{23}$ as dashed lines.

Figure 14 shows the one-dimensional resolution on the measurement of $\delta$ (see $[13,58]$ for details) for the three beam combinations considered: LE beam only $5 \mathrm{yr}$ at $1.1 \mathrm{MW}+5 \mathrm{yr}$ at $2.2 \mathrm{MW}$; the $3 \mathrm{MW}$ second maxima beam only, and the $10 \mathrm{yr}$ of DUNE running assuming the

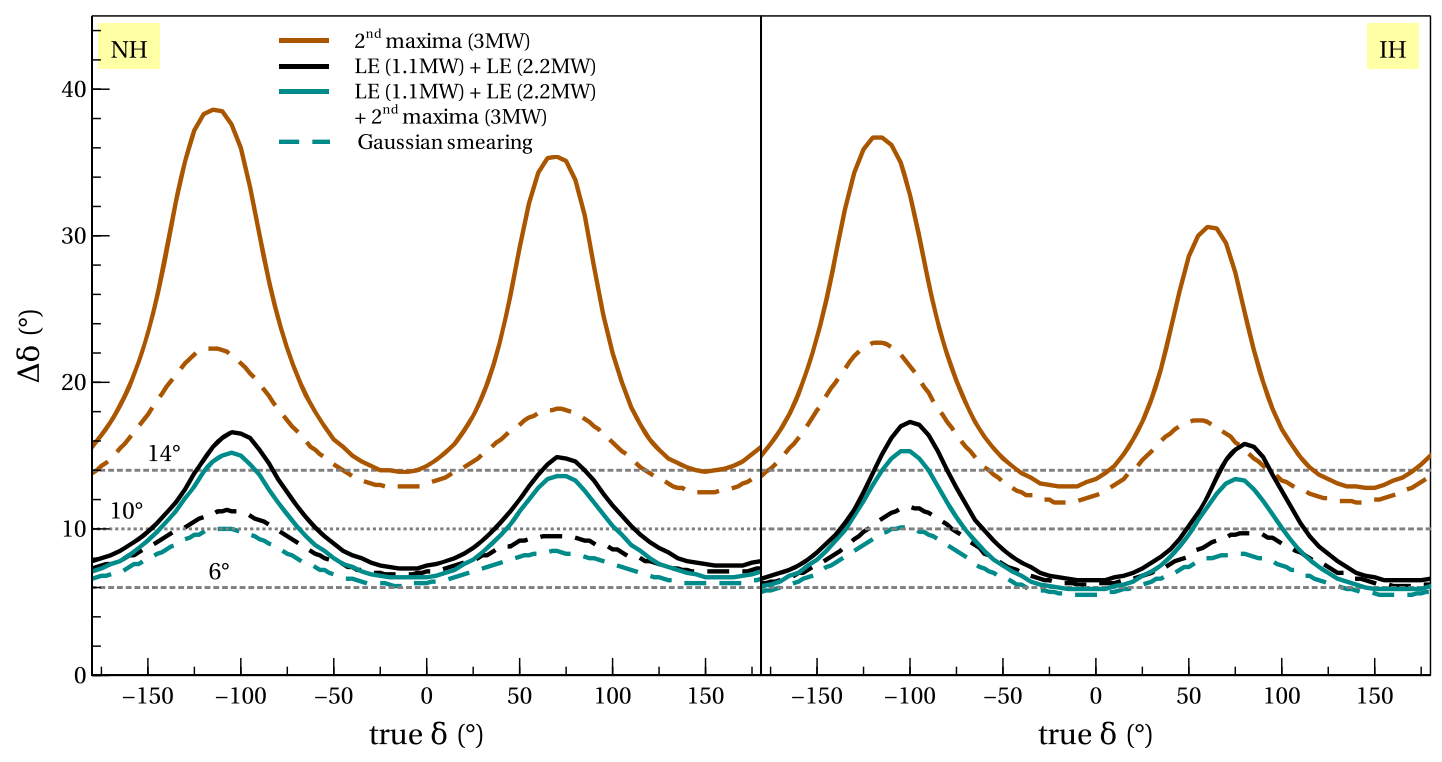

FIG. 14. Resolution on the measurement of $\delta$ as a function of the true value of $\delta$ for various beam combinations and with improved neutrino energy resolution (Gaussian smearing). 
3 MW second maxima beam runs concurrently with the 2.2 MW LE beam for five of the 10 years. Running with the 3 MW second maxima beam in conjunction with standard LE beam running at DUNE leads to some improvement in $\delta$ resolution for almost all true values of $\delta$, irrespective of the choice of hierarchy. Improved energy reconstruction capabilities lead to significantly better $\delta$ resolution, especially near the maximal $C P$-violating values. It is worth pointing out that, with the considered beam combination and the improved neutrino energy resolution, $\delta$ can be resolved within $6^{\circ}-10^{\circ}$ for all values of $\delta$. The resolution of the $C P$ phase at $\delta=\pi / 2$ lies between $\sim 11.8^{\circ}$ and $14.9^{\circ}\left(\sim 8^{\circ}\right.$ and $9.6^{\circ}$ ) for nominal (improved) detector resolution. It can be noted that the second maxima beam has a bigger impact on $\delta$ resolution for the case of IH. At $\delta=0$, we obtain $\Delta \delta \sim 5.9^{\circ}-7.5^{\circ}\left(\sim 5.5^{\circ}-7.1^{\circ}\right)$ with nominal (improved) detector resolution.

It is important to note that our inferences pertaining to the $C P$-violating phase $\delta$ in neutrino oscillations depend on the specific parameterization of the mixing matrix [Eq. (1)]

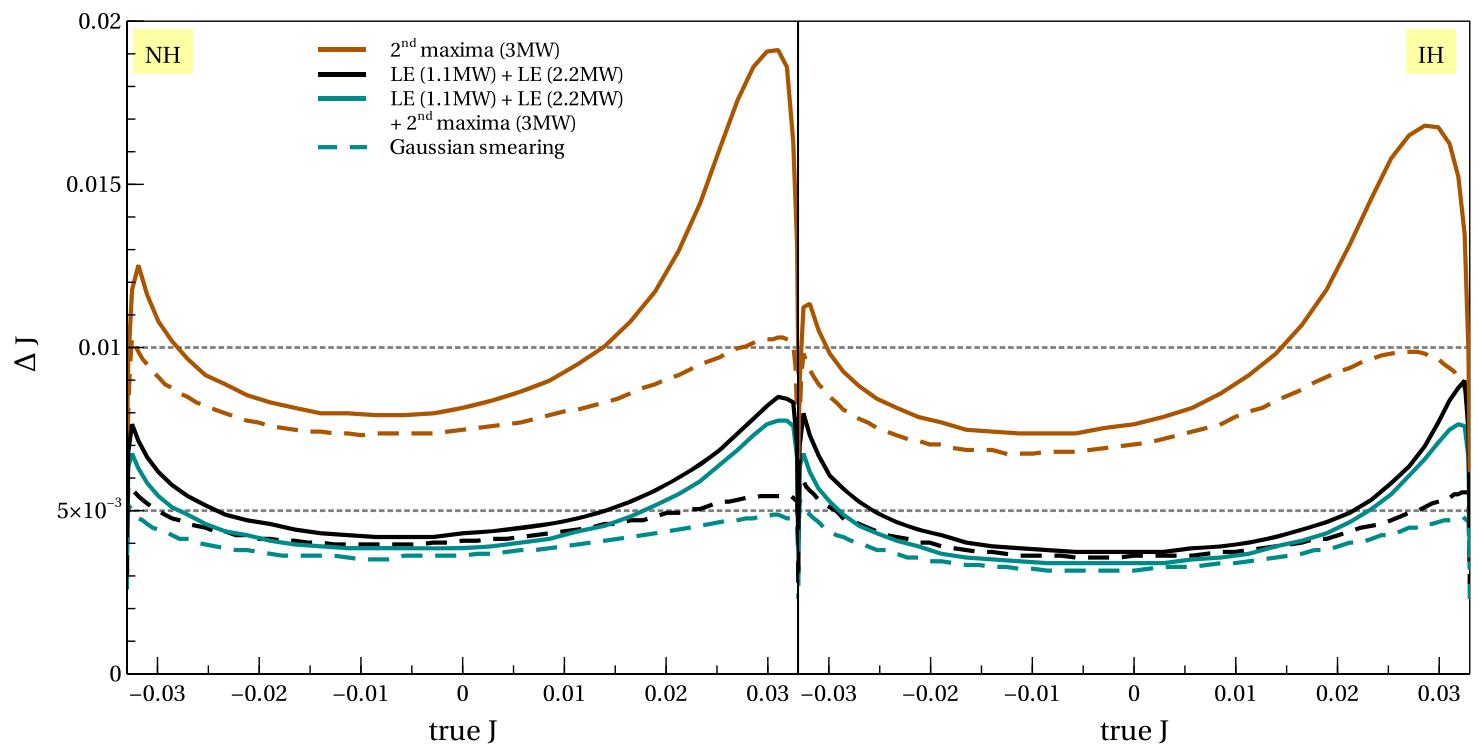

FIG. 15. Resolution on the measurement of the Jarlskog factor $J$ as a function of the true value of $J$ for various beam combinations and with improved neutrino energy resolution (Gaussian smearing).

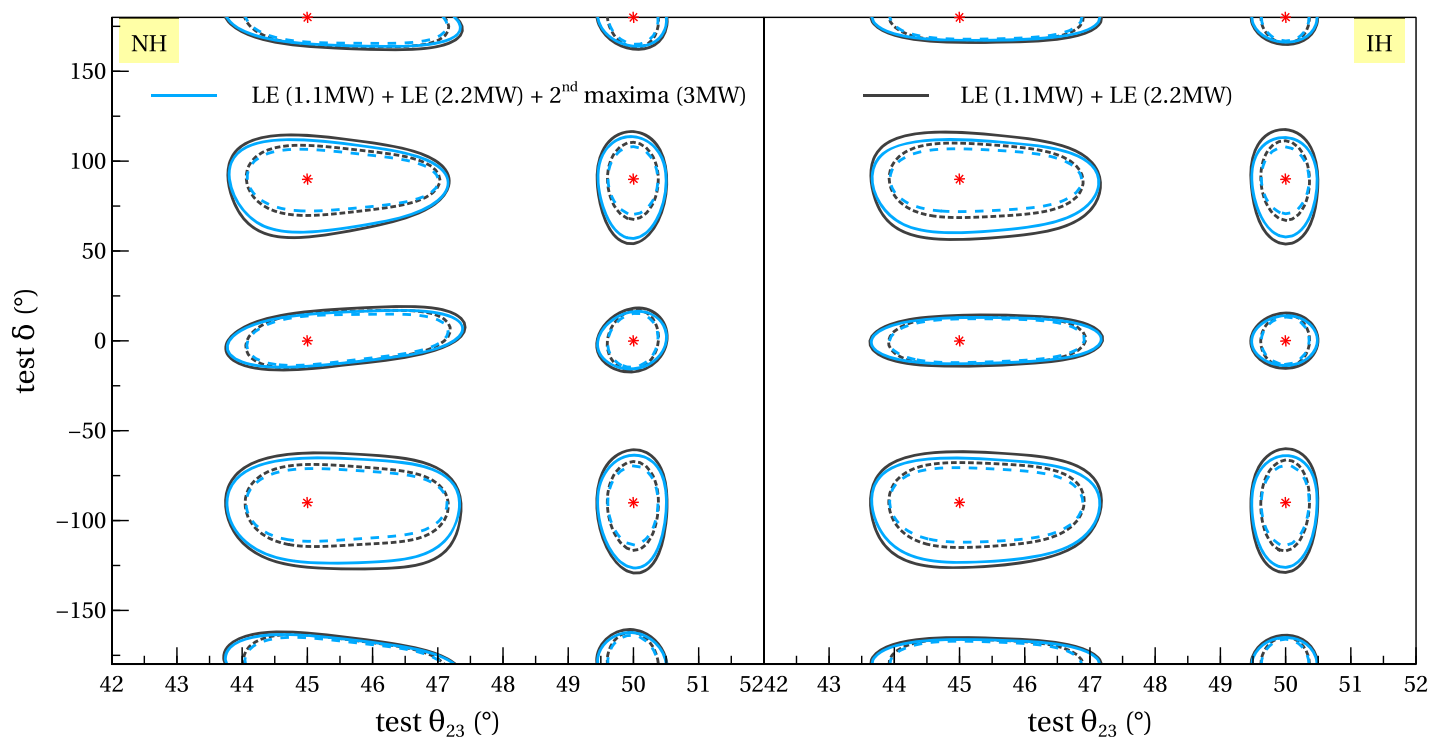

FIG. 16. $1 \sigma$ contour plots from a two-dimensional fit for $\theta_{23}$ and $\delta$ for the different beam tune combinations with DUNE configuration files (solid lines) and improved neutrino energy resolution (dashed lines). Fit results for various possible true values of $\delta=0, \pm \pi / 2, \pi$ are shown. 
and different parameterizations could result in a completely different value of $\delta$ [59]. However, the Jarlskog factor is independent of any parameterization or phase convention and, hence, an invariant. The resolution on the measurement of the Jarlskog invariant $J$ is shown in Fig. 15. Additional running with the second maxima beam using the default neutrino energy smearing produces very modest improvements to the resolution of $J$ over all values. By contrast, improvements in the neutrino energy resolution produce significant improvements in $\Delta J$ at values of $J$ near 0.033 . The combination of running in the second maxima beam and improved energy resolution for $10 \mathrm{yr}$ of DUNE operation (assuming $8 \mathrm{GeV}$ at $3 \mathrm{MW}$ runs concurrently with $80 \mathrm{GeV}$ at $2.2 \mathrm{MW}$ with the PIP-III SRF linac option) could yield a resolution of $5 \times 10^{-3}$ or better for all values of $J$.

The $1 \sigma$ contours from a two-dimensional fit to $\theta_{23}$ and $\delta$ with external constraint on $\theta_{13}$ for the combinations considered in the present work are shown in Fig. 16. Improved energy reconstruction capabilities lead to shrinking of these contours. Additionally, data from the LE and second maxima beam place independent constraints on other neutrino-oscillation parameters as well.

\section{CONCLUSION}

The present study highlights the theoretical importance of the second oscillation maximum in the $\nu_{\mu} \rightarrow \nu_{e}$ oscillation channel and its impact on addressing the current unknowns in neutrino oscillations. The international accelerator-based long-baseline neutrino program aims to constrain the parameters of the mixing matrix (or find deviations from the standard paradigm) through extremely precise measurements of flavor oscillations with systematic uncertainties well within the percent level. The idea of exploring physics at the second (and higher) maxima of $P_{\mu e}$ in the context of various experiments is of paramount importance, as it allows for a complete underpinning of the standard three-flavor oscillation framework-particularly the unknown $C P$-violating phase-since it allows us to study a wide $L / E$ range with fixed baseline experiments.

In recent times, there has been an upsurge in activity relating to analyzing the role of the second oscillation maximum in the context of $\mathrm{ESS} \nu \mathrm{SB}$ (which covers the second oscillation maximum using a $2 \mathrm{GeV} 5 \mathrm{MW}$ proton linac as a neutrino source and a water Cherenkov detector placed at $\sim 500 \mathrm{~km}$ away) and T2HKK both within and beyond the standard three-flavor mixing paradigm [16-22, 26,27]. The prospect of precision measurement of $\delta$ at MOMENT $(L=150 \mathrm{~km})$ has also been studied in Refs. [23,24]. In addition, the T2HKK [25] proposal measures the first and the second oscillation maxima with two detectors located at different sites. Therefore, it is timely to carry out a comprehensive study investigating the role of the second oscillation maximum in the context of DUNE.
The usefulness of observing the second oscillation maximum can be understood in terms of an argument based on $C P$ asymmetries. In order to observe a signal for $C P$ violation in the leptonic sector, one needs to measure the $C P$ conjugate channels $\left(\nu_{\mu} \rightarrow \nu_{e}\right.$ and $\left.\bar{\nu}_{\mu} \rightarrow \bar{\nu}_{e}\right)$. The interference term has the $C P$-violating parameter $\delta$ and should be larger (ideally) compared to the other two terms (solar and atmospheric). For the measured value of $\theta_{13}$, the interference term is large compared to the other two terms. The $C P$ asymmetry is larger at the second oscillation maximum, since $A_{\mu e}^{C P} \simeq 0.75 \sin \delta($ at $L / E \simeq 1500 \mathrm{~km} / \mathrm{GeV})$ and $A_{\mu e}^{C P} \simeq 0.3 \sin \delta$ (at $\left.L / E \simeq 500 \mathrm{~km} / \mathrm{GeV}\right)$. This implies significantly higher sensitivity to observe $C P$ violation. Now, if neutrino energy is held fixed, the baseline has to be about 3 times larger than that required for the first oscillation maximum. But this leads to a reduction in statistics by an order of magnitude. However, the other potentially viable option is to use a very intense neutrino beam from a multimegawatt proton beam. Our experimental setup comprises of LBNF/DUNE using a neutrino beam covering the second maxima in conjunction with the standard $C P$ optimized wide-band beam currently under design [8]. The neutrino beam at the second maxima is generated using a $3 \mathrm{MW}, 8 \mathrm{GeV}$ proton beam which could be realized by the PIP-III SRF linac option [28]. We note that the default $C P$-optimized wide-band beam for DUNE already covers a significant portion of the second oscillation maximum and offers the capability of precision measurement using the shape of the observed oscillation over a wide range of $L / E$. The addition of the second maxima beam running enhances the flux of neutrinos at the second maxima without the penalty of the background feed down from higher-energy neutrinos (in particular, the backgrounds from $\mathrm{NC}$ and $\nu_{\tau}$ ).

In Table VI, we list the expected improvement in sensitivity to the different unknowns with the beam and run time combinations (a) and (c) mentioned in Sec. IV. We summarize the main results of our sensitivity studies contained in Figs. 10-16 as follows.

(i) Using the default DUNE configuration files [51] and adding the second maxima beam running concurrently with the LE $2.2 \mathrm{MW}$ beam (PIP-III SRF linac option), we find a modest improvement in sensitivity to $C P$ violation, $\mathrm{MH}$, and the octant of $\theta_{23}$ (see Figs. 10-13). As can be seen from Fig. 14, the considered beam combination leads to a modest improvement in $\delta$ resolution of $\sim 1^{\circ}-2^{\circ}$ in the vicinity of maximal CP-violating values $(\delta= \pm \pi / 2)$ for the case of $\mathrm{NH}$. For IH, we get slightly larger improvement in $\delta$ resolution $\sim 3^{\circ}$ at $\delta=\pi / 2$.

(ii) Improved energy reconstruction capabilities lead to significantly better sensitivities to $C P$ violation, $\mathrm{MH}$, and the octant of $\theta_{23}$ (see Figs. 10-13) for values of $\delta$ near maximal. The improved resolution of $\delta$ from improved energy reconstruction significantly 
TABLE VI. Expected sensitivity to the different unknowns with the considered beam combinations (a) and (c) mentioned in Sec. IV. We consider a run time of $5 \mathrm{yr}$ in LE, $1.1 \mathrm{MW}$ and $5 \mathrm{yr}$ in LE, 2.2 MW distributed equally in neutrino and antineutrino modes for beam option (a). We consider a run time of $5 \mathrm{yr}$ in LE, 1.1 MW, $5 \mathrm{yr}$ in LE, 2.2 MW and $5 \mathrm{yr}$ with second maxima, $3 \mathrm{MW}$ distributed equally in neutrino and antineutrino modes for beam option (c). The numbers in parentheses correspond to the scenario with improved energy reconstruction capabilities for the considered beam combinations.

\begin{tabular}{|c|c|c|c|c|}
\hline \multirow[b]{2}{*}{ Sensitivity to } & \multicolumn{2}{|c|}{ (a) LE, $1.1 \mathrm{MW}+\mathrm{LE}, 2.2 \mathrm{MW}$} & \multicolumn{2}{|c|}{ (c) LE, 1.1 MW + LE, 2.2 MW + second maxima, $3 \mathrm{MW}$} \\
\hline & $\mathrm{NH}$ & $\mathrm{IH}$ & $\mathrm{NH}$ & $\mathrm{IH}$ \\
\hline $\begin{array}{l}\text { (i) } C P \text { violation } \\
{[\sigma] \text { at } \delta=\pi / 2}\end{array}$ & $6.9(8.9)$ & $8.5(9.9)$ & $7.6(9.9)$ & $9.0(10.8)$ \\
\hline $\begin{array}{l}\text { (ii) } C P \text { fraction } \\
f(\geq 3 \sigma) \\
f(\geq 5 \sigma)\end{array}$ & $\begin{array}{l}0.74(0.75) \\
0.54(0.58)\end{array}$ & $\begin{array}{l}0.78(0.79) \\
0.61(0.64)\end{array}$ & $\begin{array}{l}0.77(0.78) \\
0.59(0.63)\end{array}$ & $\begin{array}{l}0.80(0.81) \\
0.65(0.68)\end{array}$ \\
\hline $\begin{array}{l}\text { (iii) MH } \\
{\left[\sqrt{\Delta \chi^{2}}\right] \text { at } \delta=\pi / 2}\end{array}$ & $15.0(18.9)$ & $25.6(27.5)$ & $17.0(21.7)$ & $26.9(29.3)$ \\
\hline $\begin{array}{l}\text { (iv) Octant of } \theta_{23} \\
{\left[\Delta \chi^{2}\right] \text { at } \theta_{23}=48.8^{\circ}}\end{array}$ & $30.8(39.3)$ & $31.8(39.0)$ & $33.2(40.8)$ & $32.8(40.3)$ \\
\hline $\begin{array}{l}\text { (v) } \delta \text { resolution } \\
{\left[{ }^{\circ}\right] \text { at } \delta=0} \\
{\left[{ }^{\circ}\right] \text { at } \delta=\pi / 2}\end{array}$ & $\begin{aligned} & 7.5(7.1) \\
& 13.3(9)\end{aligned}$ & $\begin{array}{l}6.5(6.3) \\
14.9(9.6)\end{array}$ & $\begin{aligned} 6.7(6.3) \\
11.8(8)\end{aligned}$ & $\begin{array}{r}5.9(5.5) \\
11.9(8)\end{array}$ \\
\hline $\begin{array}{l}\text { (vi) } J \text { resolution } \\
{\left[\times 10^{-3}\right] \text { at } J=0} \\
{\left[\times 10^{-3}\right] \text { at } J=0.033}\end{array}$ & $\begin{array}{l}4.3(4.1) \\
4.0(2.7)\end{array}$ & $\begin{array}{l}3.7(3.6) \\
4.5(2.8)\end{array}$ & $\begin{array}{l}3.9(3.6) \\
3.6(2.4)\end{array}$ & $\begin{array}{l}3.4(3.2) \\
3.6(2.4)\end{array}$ \\
\hline
\end{tabular}

outperforms the gains from running in the second maxima beam in the vicinity of maximal $C P$-violating values (see Fig. 14).

(iii) Figure 10 depicts the sensitivity to $C P$ violation as a function of $\delta$. The $C P$-violation sensitivity at $\delta=\pi / 2$ approaches $\sim 6.9-9 \sigma \quad(\sim 8.9-10.8 \sigma)$ for nominal (improved) detector resolution.

(iv) Figure 11 shows the sensitivity to $C P$ violation as a function of the fraction of values of $\delta$ for which a given significance could be achieved. The $3 \sigma(5 \sigma)$ discovery of $C P$ violation can be achieved for $\sim 74 \%-80 \%(\sim 54 \%-65 \%)$ values of the $C P$ phase for nominal detector resolution. With improved energy reconstruction capabilities, $3 \sigma(5 \sigma)$ discovery of $C P$ violation can be achieved for $\sim 75 \%-81 \%$ $(\sim 58 \%-68 \%)$ values of the $C P$ phase.

(v) Figure 12 depicts the sensitivity to $\mathrm{MH}$ as a function of $\delta$. We note that, for $\delta=\pi / 2$, the $\sqrt{\Delta \chi^{2}}$ corresponding to the MH sensitivity lies within $\sim 15-26.9(\sim 18.9-$ 29.3) for nominal (improved) detector resolution.

(vi) The sensitivity of determining the octant of $\theta_{23}$ as a function of the true value of $\theta_{23}$ for different beam combinations is shown in Fig. 13 for $\mathrm{NH}$ and IH. A $5 \sigma$ determination of the octant of $\theta_{23}$ will be possible for at least $90 \%$ of true values of $\delta$ for $42.5^{\circ}<\theta_{23}<$ $49^{\circ}$ for $\mathrm{NH}$. We note that, for $\theta_{23}=48.8^{\circ}$, the $\Delta \chi^{2}$ corresponding to the octant sensitivity lies within 30.8-33.2 ( 39-40.8) for nominal (improved) detector resolution. (vii) One of our key results pertains to the improvement in $\delta$ resolution. It is shown in Fig. 14 that $\delta$ can be resolved better than $\sim 10^{\circ}$ for all values of $\delta$ with improved energy resolution and additional running with the second maxima beam. The resolution of the $C P$ phase at $\delta=\pi / 2$ lies between $\sim 11.8^{\circ}$ and $14.9^{\circ}\left(\sim 8^{\circ}\right.$ and $9.6^{\circ}$ ) for nominal (improved) detector resolution. It can be noted that the second maxima beam has a bigger impact on $\delta$ resolution for the case of $\mathrm{IH}$. At $\delta=0$, we obtain $\Delta \delta \sim 5.9^{\circ}-7.5^{\circ}\left(\sim 5.5^{\circ}-7.1^{\circ}\right)$ with nominal (improved) detector resolution.

(viii) We also deduce implications on the measurement of the Jarlskog invariant $J$ which is a parameterizationindependent quantity. The improvement on the resolution on the Jarlskog invariant $J$ as a function of $J$ due to improved energy resolution and additional running in the second maxima beam is depicted in Fig. 15. Additional running with the second maxima beam using the default neutrino energy smearing produces very modest improvements to the resolution of $J$ over all values. By contrast, improvements in the neutrino energy resolution produce significant improvements in $\Delta J$ at values of $J$ near 0.033 (corresponding to $\delta=\pi / 2$ ). $\Delta J \simeq 3.6-4.5 \times 10^{-3}$ $\left(\sim 2.4-2.8 \times 10^{-3}\right)$ for nominal (improved) detector resolution at $\delta=\pi / 2$ irrespective of the hierarchy. $\Delta J \simeq 3.4-4.3 \times 10^{-3}\left(\sim 3.2-4.1 \times 10^{-3}\right)$ for nominal (improved) detector resolution at $\delta=0$. The combination of running in the second maxima beam and 
improved energy resolution for $10 \mathrm{yr}$ of DUNE operation (assuming $8 \mathrm{GeV}$ at $3 \mathrm{MW}$ runs concurrently with $80 \mathrm{GeV}$ at $2.2 \mathrm{MW}$ with the PIP-III SRF linac option) could yield a resolution of $5 \times 10^{-3}$ or better for all values of $J$.

(ix) Finally, we show the $1 \sigma$ contours from a twodimensional fit to $\theta_{23}$ and $\delta$ for the beam tune combinations considered in the present work (see Fig. 16) both with DUNE configuration files [51] and with improved energy reconstruction capabilities.

We conclude that the default wide-band $C P$-optimized beam for DUNE already offers an excellent opportunity to probe physics in the vicinity of the second oscillation maxima. Improved neutrino energy resolution-coupled with very low uncertainties on the energy reconstructionis needed to fully realize this opportunity. The improved neutrino energy resolution could be achieved with further improvements in LArTPC reconstruction based on the latest DUNE studies and MicroBooNE data. The PIP-III upgrade with a pulsed SRF linac offers the opportunity for a multimegawatt $8 \mathrm{GeV}$ beam to DUNE with flux covering only the region of the second oscillation maxima, thus providing an opportunity for increasing the flux at the second maxima without the additional background from feed down from higher-energy neutrinos. Such a beam, running concurrently with the $2.2 \mathrm{MW} 80 \mathrm{GeV}$ beam from
PIP-III, can further improve sensitivity to the three-flavor oscillation parameters, but we find the gain is modest.

\section{ACKNOWLEDGMENTS}

It is a pleasure to thank Peter Denton for insightful discussions and Enrique Fernandez-Martinez for email communication in connection with Ref. [15] after the preprint appeared on the arXiv. We acknowledge the use of HPC cluster at SPS, JNU funded by DST-FIST for numerical computations. J.R. and S.S. thank the University Grants Commission for financial support in the form of research fellowships. This material is based upon work supported by the U.S. Department of Energy, Office of Science, Office of High Energy Physics under Contract No. DE-SC0012704; the Indian funding from University Grants Commission under the second phase of University with Potential of Excellence (UPE II) at JNU and Department of Science and Technology under DSTPURSE at JNU. This work has received partial funding from the European Union's Horizon 2020 research and innovation program under the Marie Skodowska-Curie Grant Agreement No. 690575 and No. 674896. P. M. thanks the particle physics group at Brookhaven National Laboratory for the warm hospitality during the early stages of this work.
[1] B. Pontecorvo, Sov. Phys. JETP 26, 984 (1968), http://www .jetp.ac.ru/cgi-bin/e/index/e/26/5/p984?a=list .

[2] T. Kajita and A. B. McDonald, For the discovery of neutrino oscillations, which shows that neutrinos have mass, the Nobel Prize in Physics 2015.

[3] P. F. de Salas, D. V. Forero, S. Gariazzo, P. Martínez-Miravé, O. Mena, C. A. Ternes, M. Tórtola, and J. W. F. Valle, J. High Energy Phys. 02 (2021) 071.

[4] I. Esteban, M. Gonzalez-Garcia, M. Maltoni, T. Schwetz, and A. Zhou, J. High Energy Phys. 09 (2020) 178.

[5] C. H. Albright and M.-C. Chen, Phys. Rev. D 74, 113006 (2006).

[6] M. Fukugita and T. Yanagida, Phys. Lett. B 174, 45 (1986).

[7] C. Adams et al. (LBNE Collaboration), arXiv:1307.7335.

[8] R. Acciarri et al. (DUNE Collaboration), arXiv:1512.06148.

[9] R. Acciarri et al. (DUNE Collaboration), arXiv:1601.05471.

[10] M. Bass et al. (LBNE Collaboration), Phys. Rev. D 91, 052015 (2015).

[11] M. Bishai, M. Diwan, S. Kettell, J. Stewart, B. Viren, E. Worcester, R. Tschirhart, and L. Whitehead, in Proceedings, 2013 Community Summer Study on the Future of U.S. Particle Physics: Snowmass on the Mississippi (CSS2013): Minneapolis, MN, USA, 2013 (2013) [arXiv:1307.0807].

[12] X. Qian, J. J. Ling, R. D. McKeown, W. Wang, E. Worcester, and C. Zhang, in Proceedings, 2013 Community Summer
Study on the Future of U.S. Particle Physics: Snowmass on the Mississippi (CSS2013): Minneapolis, MN, USA, 2013 (2013) [arXiv:1307.7406].

[13] P. Coloma, H. Minakata, and S. J. Parke, Phys. Rev. D 90, 093003 (2014).

[14] P. Huber and J. Kopp, J. High Energy Phys. 03 (2011) 013; 05 (2011) 024(E).

[15] V. De Romeri, E. Fernandez-Martinez, and M. Sorel, J. High Energy Phys. 09 (2016) 030.

[16] E. Baussan et al. (ESSnuSB Collaboration), Nucl. Phys. B885, 127 (2014).

[17] E. Wildner et al., Adv. High Energy Phys. 2016, 1 (2016).

[18] M. Blennow, E. Fernandez-Martinez, T. Ota, and S. Rosauro-Alcaraz, Eur. Phys. J. C 80, 190 (2020).

[19] S. K. Agarwalla, S. Choubey, and S. Prakash, J. High Energy Phys. 12 (2014) 020.

[20] K. Chakraborty, S. Goswami, C. Gupta, and T. Thakore, J. High Energy Phys. 05 (2019) 137.

[21] M. Ghosh and T. Ohlsson, Mod. Phys. Lett. A 35, 2050058 (2020).

[22] M. Ghosh (for the ESSnuSB Collaboration), Proc. Sci. NuFact2019 (2020) 038.

[23] J. Cao, M. He, Z.-L. Hou, H.-T. Jing, Y.-F. Li, Z.-H. Li, Y.-P. Song, J.-Y. Tang, Y.-F. Wang, Q.-F. Wu et al., Phys. Rev. ST Accel. Beams 17, 090101 (2014). 
[24] J. Tang, S. Vihonen, and T.-C. Wang, J. High Energy Phys. 12 (2019) 130.

[25] K. Hagiwara and N. Okamura, J. High Energy Phys. 01 (2008) 022.

[26] S. Choubey, M. Ghosh, D. Kempe, and T. Ohlsson, arXiv: 2010.16334.

[27] K. Chakraborty, D. Dutta, S. Goswami, and D. Pramanik, arXiv:2012.04958.

[28] Proton Improvement Plan III (2015), https://pip3.fnal.gov/.

[29] N. None, https://www.osti.gov/biblio/1631119.

[30] J. Beringer et al. (Particle Data Group), Phys. Rev. D 86, 010001 (2012).

[31] C. Giunti, Phys. Lett. B 686, 41 (2010).

[32] A. de Gouvêa, K. J. Kelly, G. V. Stenico, and P. Pasquini, Phys. Rev. D 100, 016004 (2019).

[33] A. Ghoshal, A. Giarnetti, and D. Meloni, J. High Energy Phys. 12 (2019) 126.

[34] J. Rout, S. Roy, M. Masud, M. Bishai, and P. Mehta, Phys. Rev. D 102, 116018 (2020).

[35] W. Marciano and Z. Parsa, Nucl. Phys. B, Proc. Suppl. 221, 166 (2011).

[36] A. Cervera, A. Donini, M. Gavela, J. J. Gomez Cadenas, P. Hernandez, O. Mena, and S. Rigolin, Nucl. Phys. 579, 17 (2000).

[37] M. Freund, Phys. Rev. D 64, 053003 (2001).

[38] E. K. Akhmedov, R. Johansson, M. Lindner, T. Ohlsson, and T. Schwetz, J. High Energy Phys. 04 (2004) 078.

[39] E. K. Akhmedov, Phys. Scr. T121, 65 (2005).

[40] M. Masud, A. Chatterjee, and P. Mehta, J. Phys. G 43, 095005 (2016).

[41] C. Jarlskog, Phys. Rev. Lett. 55, 1039 (1985).
[42] C. Jarlskog, Phys. Lett. B 609, 323 (2005).

[43] S. P. Mikheev and A. Y. Smirnov, Sov. Phys. Usp. 30, 759 (1987).

[44] L. Wolfenstein, Phys. Rev. D 17, 2369 (1978).

[45] P. Huber, M. Lindner, and W. Winter, Comput. Phys. Commun. 167, 195 (2005).

[46] P. Huber, J. Kopp, M. Lindner, M. Rolinec, and W. Winter, Comput. Phys. Commun. 177, 432 (2007).

[47] A. M. Dziewonski and D. L. Anderson, Phys. Earth Planet. Inter. 25, 297 (1981).

[48] H. Nunokawa, S. J. Parke, and J. W. F. Valle, Prog. Part. Nucl. Phys. 60, 338 (2008).

[49] J. Rout, M. Masud, and P. Mehta, Phys. Rev. D 95, 075035 (2017).

[50] Dune fluxes (2017), https://home.fnal.gov/ 1jf26/ DUNEFluxes/.

[51] T. Alion et al. (DUNE Collaboration), arXiv:1606.09550.

[52] R. Acciarri et al. (DUNE Collaboration), arXiv:1601.02984.

[53] B. Abi et al. (DUNE Collaboration), arXiv:2002.03005.

[54] M. Masud and P. Mehta, Phys. Rev. D 94, 013014 (2016).

[55] X. Qian, A. Tan, W. Wang, J. J. Ling, R. D. McKeown, and C. Zhang, Phys. Rev. D 86, 113011 (2012).

[56] M. Masud and P. Mehta, Phys. Rev. D 94, 053007 (2016).

[57] E. Ciuffoli, in Part of Proceedings, Prospects in Neutrino Physics (NuPhys2016), London, UK, 2016 (2017) [arXiv: 1704.08043].

[58] P. Coloma, A. Donini, E. Fernandez-Martinez, and P. Hernandez, J. High Energy Phys. 06 (2012) 073.

[59] P. B. Denton and R. Pestes, arXiv:2006.09384. 